XII.

\title{
Fistelöffnungen \\ am oberen Pole des Trommelfells über dem Processus brevis des Hammers, deren Pathogenese und Therapie.
}

\author{
Yon \\ Dr. F. Kretsehmann \\ in Magdeburg.
}

Seitdem die Behandlung der eitrigen Entzündungen des Mittelohres eine vorzugsweise locale und chirurgische geworden ist, bat sich die Prognose dieser Erkrankungen wesentlich giunstiger gestaltet. Die zahlreichen Heilungen, welche durch die Behandlung mit kaustischer Lapislösung, durch Entfernung cariöser Herde mittelst Evidement oder Galvanokaustik erreicht sind, legen beredtes Zeugniss ab für unsere Behauptung. Souverain aber unter den chirurgischen Mitteln ist die operative Eröffnung des Warzenfortsatzes, welche, ganz abgesehen von ihrer hohen Bedeutung als lebensrettende Operation, die Ausheilung vieler langwieriger und früher als unheilbar geltender chronischer Eiterungsprocesse im Gehörorgane ermöglicht hat. ${ }^{1}$ ) Diese Resultate der Warzenfortsatzeröffnung konnten aber nur erzielt werden, nachdem durch Aufstellung bestimmter Indicationen ${ }^{2}$ ) eine richtige Auswabl der zu operirenden Erkrankungsfälle getroffen werden konnte, und nachdem durch Vervollkommnung der Operationsmethode die Gefahren, welche früher den Eingriff als äusserst bedenklich erscheinen liessen, jetzt erheblich vermindert sind. Trotzdem bleibt aber doch eine ganze Anzahl von Eiterungsprocessen, meistentheils chronischer Natur, übrig, deren Heilung höchst langwierig und unsicher ist und durch die Eröffnung des Warzenfortsatzes allein wenig oder gar nicht beeinflusst wird, ich meine jene Falle, welche mit einer Perforation der „Membrana Shrapnelli“ oder, wie es vielleicht richtiger zu sagen wäre, mit einer Perforation iiber dem kurzen Ham-

1) s. Schwartze, Dieses Arch. Bd. XIV. S. 204.

2) Ebenda S. $205 \mathrm{ff}$.

Archiv f. Ohrenheilkunde. XXV, Bd. 
merfortsatz einhergehen. $\quad$. Tröltseh ${ }^{1}$ spricht sich über die Affection folgendermaassen aus: ,Nicht selten findet sich Perforation am oberen Pole in der Membrana flaccida Shrapnelli, letztere Form erweist sich durchschnittlich sehr hartnäckig gegen die Behandlung etc." Bezold ${ }^{2}$ ) findet Perforationen im oberen Pole in $1 \frac{1 / 4}{4}$ Proc. aller Krankheitsfälle. Morpurgo ${ }^{3}$ ) berichtet, dass Prof. Mo os unter 126 Perforationen viermal den Sitz in der Membrana flaccida sab. Nach Hessler ${ }^{4}$ ) sollte auf 100 Fälle von Erkrankungen des Ohres nur einer mit Perforation der Shrapnell'sehen Haut kommen. In den Krankenjournalen der Halle'schen Ohrenklinik aus den letzten Jahren war das Vorkommen solcher Perforationen mindestens dreimal so bäufig verzeichnet. Unter 1000 Krankheitsfällen (aus dem Jahrgange 1886 entnommen) fand ich $30 \mathrm{mal}$,Fistel ïber dem Processus brevis" notirt, und ich glaube, dass diese Zahl noch zu klein ist, weil in manchen Fällen die genauere Angabe des Sitzes der Perforationen im Journal fehlt. Unter diesen 1000 Fällen waren 368 Eiterungen.

Ausser durch den eigenthümlichen Sitz der Perforationsoffnung zeichnet sich diese Form vor den ubrigen Eiterungen noch durch besondere Symptome aus.

In erster Linie ist zu erwähnen das bäufige Fehlen des Perforationsgeräusches. Nach Morpurgo ${ }^{3}$ ) war ungefähr in $3 / 4$ der beobachteten Fälle das Perforationsgeräusch nicht nachweisbar. Diese Thatsache gab die Veranlassung, bei den Formen mit Perforation der "Shrapnell'schen Membran" einen von der übrigen Paukenhöhle abgeschlossenen Raum anzunehmen, in welchem die Eiterung sich abspielt.

Bezold, Morpurgo, Hessler, welche eine eingehendere Beschäftigung mit diesem Gegenstande literarisch bethätigt haben, nehmen entweder den Prussak'schen Raum oder das von Politzer ${ }^{5}$ ) beschriebene Höhlensystem als die Oertlichkeit an, in weleher der Process seinen Anfang nimmt. Nach Prussak ${ }^{6}$ ) befindet sich zwischen Membrana Shrapnelli and Hammerhals ein Zwischenraum von $1 / 4-1 / 2$ Linie. Dieser Zwischenraum wird zu einer

\footnotetext{
1) Lehrbuch der Ohrenheilkunde. VII. Aufl. 1881. S. 456.

2) Dieses Arch. Bd. XXI. S. 239.

3) Ebenda. Bd, XIX. S. 284.

4) Ebenda. Bd. XX. S. 134.

5) Lehrbuch der Ohrenheilkunde. 1882 S. 25.

6) Dieses Arch. Bd. III. S. 265.
} 
Höhle vervollständigt, welche, nur an einer Seite offen bleibend, mit der Paukenhöhle communicirt. Die Begrenzungen der Höhle sind nach aussen, die Membrana flaccida, nach innen die laterale Seite des Hammerhalses, nach nnten die obere Fläche des kurzen Fortsatzes; die obere Wand wird dadurch gebildet, dass die Membrana flaccida, sobald sie den Margo tympanicus erreicht, ein Blatt nach innen und abwärts an die Spina capitis mallei, d. i. an die kleine knöcherne Erhabenheit sendet, welche nach aussen den Hammerhals vom Kopfe scheidet.

Die Höhle ist ziemlich klein und hat zur Basis nur die obere Fläche des kurzen Fortsatzes. Die vordere Grenze wird dureh eine Duplicatur der Tunica propria des Trommelfells gebildet, welche sich mit dem Trommelfell selbst unter stumpfem Winkel vereinigt, und welche ihrerseits die obere hintere Begrenzungsmembran der vorderen Trommelfelltasche darstellt. Sie ist ansgespannt zwischen dem Ligamentum mallei anterius (welches die durch die Glaserspalte ziehenden Gebilde enthält), vorderer Kante des Hammerhalses, vorderer Kante des Processus brevis, verschmilzt mit dem Trommelfell in einer auf- und vorwärts steigenden Linie und zieht von dem Punkte, wo diese Linie den Margo tympanicus erreicht, bis zur Insertionsstelle des Ligamentum anterius. Die obere Begrenzungsmembran muss also mit der etwas nach vorn geneigt stehenden vorderen unter einem spitzen Winkel zusammenstossen. Nach hinten zu wird die Höhle nicht begrenzt, sondern communicirt mit dem Cavam tympani.

Es würde demnach der Raum annähernd eine abgeschnittene unregelmässige Pyramide vorstellen, deren kleinste Seiten nach unten (obere Fläehe des kurzen Fortsatzes) und nach innen (Hammerhals) gelegen sind. Die Communicationsöffnung würde der Beschreibung nach begrenzt werden nach aussen durch eine Linie von der Spitze des Processus brevis, nach dem äusseren Rande der oberen Begrenzungsmembran, wenn dieselbe als mit freiem Saum nach hinten endend zu denken ist ${ }^{1}$ ), nach oben von diesem Rande selbst, nach innen von der hinteren Kante des Hammerhalses, nach unten von der hinteren Kante des Processus brevis des Hammers. Prussak wählt für diesen Raum den Namen obere Tasche.

Prof. Politzer, welcher die anatomischen Verhältnisse eben-

1) Nähere Angaben fehlen darüber. 
falls untersucht hat, stimmt im Grossen und Ganzen den Prussakschen Resultaten bei, dass iiber dem kurzen Fortsatz ein Raum existire, welcher ïber der hinteren Tröltsch'schen Tasche in die Paukenhöhle einmünde. Zuweilen constatirte er noch eine Communication mit der vorderen Tasehe. Ueber diesem Raum gelegen findet Politzer ein Höhlensystem, in welches der Rivinische Ausschnitt hereinragt. Er illustrirt dasselbe ${ }^{1}$ ) an frontalen Durchschnitten durch Gehörgang und Pankenhöhle, welche den Hammer getroffen haben. Abgesehen davon, dass dieses System einen nur sehr kleinen Raum, makroskopisch betrachtet, einnimmt, ist der Beweis für die Existenz eines allseitig begrenzten Hoblraumes durch die eine Schnittrichtung nicht gebracht, sondern es bedürte daza noch eines auf dem ersten vertical gestellten Schnittes, der dann ein ähnliches Bild maschenförmiger Räume liefern mïsste.

Nicht nnerwähnt darf hier die anatomische Darstellung bleiben, wie sie Sexton ${ }^{2}$ ) giebt, die, zwar in Manchem nicht ganz genau, doch im Wesentlichen den Kern der Sache trifft.

Der "Attic" (unter Attic verstehen die Amerikaner den Raum im Hause unmittelbar unter dem Dache) tympanicus ist ein pyramidenförmiger Recess ${ }^{3}$ ) iiber dem Atrium (eigentliche Paukenhöhle $[s a]$ ) und über dem tympanalen orificium des äusseren Gehörgangs. Die obere vordere Grenze bildet das Tegmen, die innere untere ein Vorsprung, weleher dureh die Vorwölbung des äusseren Semicircular- und Facialkanals bedingt wird. Die äussere Grenze ist die an Dicke zunehmende Platte des äusseren Gehörgangs. Der "Attic" communicirt durch eine weite Oeffnung mit dem Antrum, durch eine elliptische Oeffnung, gebildet durch den First des Facialkanals nach innen und dem Tympanalrand der Gehörgangsplatte nach aussen, mit dem Atrium. Der "Attie" ist getheilt durch Hammer und Amboss in eine äussere und innere Abtheilung. Die Knöchelchen gehören dem "Attic" nur zum Theil an. Der lange Fortsatz des Amboss und Handgriff des Hammers reichen in das Atrium herab. Die beiden Atticräume communiciren oberwärts mit einander, unten mit

1) Lehrbuch. $1882 . \mathrm{S}, 45$ u. Wiener, Medicinische Wochenschr. 1870.

2) Transactions of the American otol. society 18 ann. meet. Vol. III. p. 4.

3) Der als „Attic“ geschilderte Raum wird von Schwalbe: "Die Lehre von den Sinnesorganen", in Hoffmann's "Lehrbuch der Anatomie des Menschen." II. Auflage. 1887. S. 464 passender mit Recessus epitympanicus bezeichnet. 
dem Atrium (nicht constant), vorn mit der Tuba (?), hinten mit dem Antrum. Das äussere Stiick ist keilförmig, weiter oben und nach unten zu sich verjüngend, so dass Körper des Amboss und Hals des Hammers beinahe in Contact (?) sind mit der Gehörgangsplatte.

Die Resultate, welche wir beziglich der anatomischen Verhältnisse dieser Theile gewonnen haben, weichen von den erwähnten etwas $a b .^{1}$ )

Betrachten wir die laterale Paukenwand von der Innenseite, so finden wir sie gebildet einmal durch das Trommelfell und zweitens durch eine Knochenwand, welche der Schläfenbeinschuppe angehört (Fig. 1). Dieselbe hat eine annähernd kreisbogenförmige Gestalt. Der untere Rand dieser Knochenwand ist gebildet durch den Margo tympanicus, welcher sich an seiner höchsten Stelle zum Rivinischen Ausschnitt erweitert. Es bildet diese Wand mit der Trommelfellebene einen stumpfen, nach aussen offenen Winkel, an welchem der Rivinische Ausschnitt ebenfalls Theil nimmt, so dass also auch er, resp. eine durch ihn gelegte Ebene zur Trommelfellebene geneigt stehen wïrde.

Diese Knochenwand,

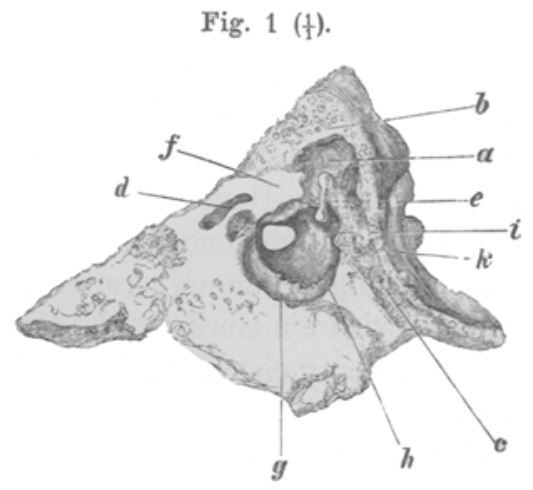

Laterale Wand der Paukenhöhle, Labyrinthwand bis auf das Stuck bei $f$ entfernt: $a$ halbkreisförmige Knochenniesehe der Sohlaffensohuppe. $b$ Bruehstelle des entfernten Tegmen tympani. $c$ knöcherne Tuba, eröfnet. $d$ erOffneter Facialkanal, e kurzer Hebelarm des in Fig. 11 abgebildeten Instrumentes zur Entfernung des Amboss. $f$ Rest der Labyrinthwand. $g$ Trommelfellrest. $h$ äusserer Gehörgang. $i$ Riviniseher Aussehnitt. $k$ Spina tympanica major. welche über dem äusseren Gehörgang gelegen ist, geht nach oben zu mit ziemlich starker Biegung in das Tegmen tympani, nach vorn in gleicher Weise in die vordere Paukenhöhlenwand über. Nach hinten zu erfolgt

1) Als ich die anatomischen. Untersuchungen zum Abschluss gebracht hatte, erschien das Lehrbuch der Anatomie der Sinnesorgane von $\mathrm{Schwalbe.}$ Obwohl in demselben grösstentheils die Verhältnisse so geschildert sind, wie ich sie ebenfalls fand, gebe ich doch meine Resultate ausführlich wieder, da einige Punkte, auf die es mir wegen der praktischen Nutzanwendung ankam, näher berührt werden mussten. 
der Uebergang ganz allmählich und gleichmässig in die Iaterale Begrenzungskante des Aditus ad antrum 1). Von innen her betrachtet, haben wir also eine Niesche vor uns, welche über dem Gehörgang liegt und mit dem Antrum communicirt. Die nach der Paukenhöhle zu gelegene weite Oeffnnng wird durch Hammer und Amboss zum Theil verschlossen.

Der Hals des Hammers setzt an den Handgriff in einem Winkel ron $150^{\circ}$ (Hy rt1), (nach eigenen Messungen 140 ) an. Dadurch divergiren Kopf and Hals um eben diesen Winkel vom Trommelfell, in welches der Handgriff befestigt ist, und in noch höherem Grade von der oben erwähnten Knochenwand, welche mit der Trommelfellebene einen Winkel bildet. An seiner hinteren Fläche articulirt der Hammerkopf mit dem Amboss. Es muss daher auch dieser Knochen einen gewissen Abstand (1 1/2 Mm.) von der lateralen Paukenhöhlenwand haben. Die Richtung des abgeplatteten Ambosskörpers wird bestimmt durch seine Gelenkverbindung mit dem Hammer und entspricht einer etwas lateralwärts geneigten Sagittalebene. Durch die soeben beschriebenen knöchernen Theile (Hammer, Amboss, laterale Pankenwand, Tegmen tympani) wird ein Gerïst gebildet, das durch membranöse Ueberbrückung der meisten Zwischenräume zu einem Hohlraum vervollständigt wird.

Nach Helmholtz ${ }^{2}$ ) legt sich der Hammerhals dicht an die Spina tympanica major an und wird dort durch eine straffe Bandmasse, das Ligamentum mallei anterius festgehalten. Von der Crista am Halse des Hammers verlänft ein anderer Bandzug ${ }^{3}$ ) fächerförmig an das Os temporum, welcher theilweise den von diesem Knochen lateral und Hammer nebst Amboss medial begrenzten, längsovalen Spalt ausfüllt. Die übrig bleibenden Lüicken werden in der Regel durch eine Membran geschlossen, welche vom Ligamentum anterius beginnend in horizontaler Richtnng bis zur Insertion des kurzen Ambossschenkels an die Paukenwand verläuft (Fig. 2).

Helmholtz ${ }^{4}$ ) beschreibt, vom Ligamentum anterius ausgehend, 3 Schleimhautfalten, eine nach oben und zwei nach unten

1) Mit Aditus ad antrum bezeichne ich die dreieckige Oeffnung, welche Pauken- und Warzenfortsatzhöhle verbindet (vgl. Fig. 7, S. 175).

2) Die Mechanik der Gehörknöchelchen und des Trommelfells. Separatabdruck aus Pflüger's Archiv für Physiologie. I. Jahrg. S. 18.

3) 1. c. Fig. 5. S. 21.

4) 1. c. S. 19 . 
zu verlaufend. „Nach oben zu zieht sich die Falte ungefähr längs der Contourlinie des Knochens (Hammers) hin, immer schmal und sichelförmig bleibend, da hier die äussere Wand der Trommelhöhle dem Kopf des Hammers überall sehr nahe bleibt. Endlich endet diese Schleimhantfalte oben auf dem Kopfe und in ibrem Rande liegt das kurze rundliche Ligamentum mallei superius."

Ibr anderer Insertionsrand befindet sich an der Stelle, wo die seitliche in die vordere Paukenhöhlenwand übergeht. Die Ebene, in welcher diese Membran verläuft, hält ungefähr die Mitte zwischen einer sagittalen und frontalen. Es schliesst diese Falte unseren Raum nach vorn und medialwärts ab. Die Insertion derselben an der am meisten nach vorn gelegenen Linie des Hammerkopfes bringt es mit sich, dass die laterale Seite dieses Knochens dem Innern des Hohlraumes angehört. Nach unten verlängert sich das Ligamentum anterius, wie Helmboltz sagt, in zwei Schleimhautfalten. „Die eine läuft von der Wurzel des

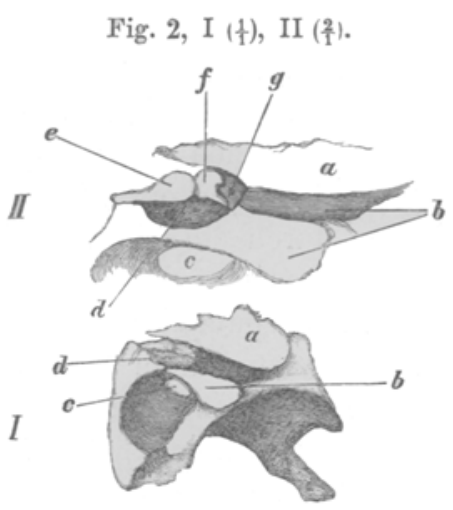

Obere Gehörgangswand und Schuppentheil grösstentheils entfernt. $a$ Tegmen tympani. $b$ Sägefläche der oberen Gehörgangswand, $c$ äusserer Gehörgang. $d$ Membran zwischen Hammer, Amboss und Schläfenwand. $e$ Amboss. $f$ Hammer. $g$ Ligamentum anterius.

Processus Folianus gegen die Spitze des kurzen Fortsatzes hin. Ibre gegenüberliegende Ansatzlinie liegt am Trommelfell. Es ist dies die Falte, welche die vordere und hintere Trommelfelltasche von einander seheidet, so dass der Raum über dem Processus brevis hauptsächlich der hinteren Tasche zufällt." Nach den Untersuchungen von Helmholtz wäre eine obere Tasche, wie Prussak sie beschreibt, nicht vorhanden. Ich habe in einer Anzahl von Präparaten die Verhältnisse in der Weise gefunden, wie sie Helmboltz schildert, ebenso oft aber auch so, wie Prussak angiebt. Die letzterwähnte Falte ist identisch mit der von Prussak als hintere obere Begrenzungsmembran der vorderen Tasche beschriebenen Duplicatur des Trommelfells, welche den Raum zwischen vorderer Kante des kurzen Fortsatzes, Hammerhals, Ligamentum anterius und Trommelfell ausfüllt (an letzterem in einer Linie, welche von der Spina major zur Spitze des 
Processus brevis verläuft) und zugleich als vordere Begrenzungsmembran seiner oberen Tasche dient.

Die zweite Helmholtz'sche Verlängerung des Ligamentum anterius nach unten ist eine schmale Falte mit freiem Rande, welche sich an der unteren Kante des Processus Folianus an dem Hammerhals entlang zur Sehne des Trommelfellspanners hinzieht. Sie ist wohl ein Theil jener Membran, welche sich zwischen Sehne des Tensor tympani, Semikanal für diesen Muskel und unterer Kante der mit dem Ligamentum anterius verlaufenden Gebilde ausspannt und nach vorn über dem oberen Rand des Ostium tympanicum tubae inserirt. Von oben gesehen bietet diese Membran das Ansehen einer Kuppel. ${ }^{1}$ )

Der Hammerkopf ist, wie oben bereits angedeutet, an das Tegmen durch ein Band befestigt, das Ligamentum mallei superius, welches von der die vordere mediale Begrenzungswand bildenden Schleimhautfalte eingeschlossen wird. Diese Falte setzt sich in vielen Fällen noch auf den Ambosskörper fort, wo sie dann als Ligamentum incudis ( $\mathrm{Henle}$ ) mit freiem Rande endet. Am Tegmen tympani findet sich eine sagittal verlaufende Leiste, an welche sich diese Schleimhautfalte ansetzt. Durch diese Falte, sowie durch den Hammerkopf und Ambosskörper wird die mediale Wand des zu beschreibenden Raumes gebildet. Als obere dient das Tegmen tympani. Eine hintere Wand existirt nicht, da hier sich die Oeffnung des Aditus ad antrum befindet. Medialwärts bleibt eine Lücke, welche die Communication mit der Paukenhöhle vermittelt. Sie hat ungefähr die Gestalt eines Trapezoides. Der vordere Rand wird gebildet durch das frei endende Ligamentum incudis, resp. wenn dieses fehlt, durch das Ligamentum mallei superius, der untere durch die obere Kante des kurzen Ambossfortsatzes, der obere durch das Tegmen tympani und der hintere würde einer den Aditus ad antrum in zwei Hälften theilenden Linie entsprechen. Nicht selten verengt eine Membran, welche in dem Winkel, den der kurze Ambossschenkel mit der Paukenwand bildet, eingelassen ist, die Communicationsöffnung, wie Figur 3 zeigt.

Die den Spalt zwischen Sehuppenwand einerseits, Ambossschenkel, Körper, Hammerhals andererseits ausfüllende Membran inserirt lateralwärts von hinten beginnend an der Stelle, wo der kurze Ambossschenkel mit der hinteren Paukenwand articulirt, ver-

1) Prussak 1.c. 
läuft dann in horizontaler Richtung an der inneren Schuppenwand, bis sie den Margo tympanicus erreicht ( $3 \mathrm{Mm}$. von der höchsten Spitze des Rivinischen Ausschnittes entfernt). Sie setzt nun quer über diesen Ausschnitt und verschmilzt auf diesem Wege mit der Membrana flaccida. An der vorderen Kante der Incisura Rivini geht sie in das Ligamentum anterius über. Die Verschmelzung mit der Shrapnell'schen Membran geht in derselben Höhe und zum Theil in derselben Linie vor sich, in welcher das die hintere Tröltschsche Tasche begrenzende mediale Blatt sich mit der Membran verbindet. Dies bestätigt auch Helmholtz; wenn er sagt: „Es zweigt sich hier (an der Spina des Hammerhalses) ein zweiter Bandzug ab, der sich an den scharfen Rand des Rivinischen Ausschnittes ansetzt, indem er nach hinten der Ansatzlinie der hinteren Trommelfelltasche folgt." Medialwärts inserirt die horizontale Falte an der unteren Kante des kurzen Ambossschenkels, verläuft dann in der Einsenkung des Ambosskörpers, geht auf den Hammerhals über

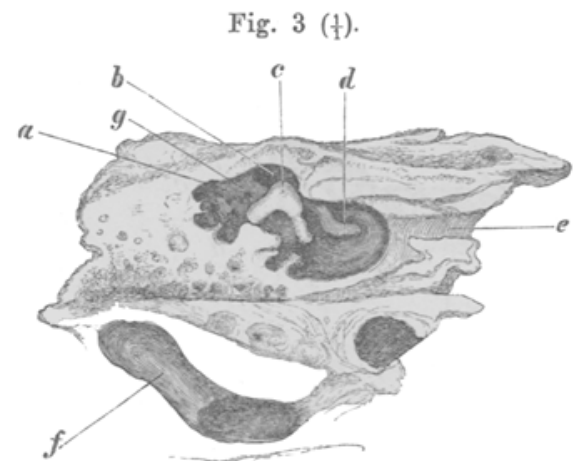

Felsenbein durch einen in der Längsaxe der Pyramide verlaufenden Sägescbnitt getheilt. Innere Ansicht der lateralen Hälfte. a Antrum mastoideum. $b$ Oeffnung, welche in den HammerAmboss-Schuppenraum führt. $c$ Amboss. $d$ Hammer. $e$ knöcherne Tuba, geöffnet. $f$ Sulcus transversus. $g$ Membran, welche die Communication zwischen Paukenhöhle und HammerAmboss-Schuppenraum znm Theil verlegt. und erreicht zuletzt das

Ligamentum anterius. Es wird durch diese Anordnung eine für unsere Betrachtungen zwar nicht wesentliche, dennoch aber erwähnenswerthe Tasche gebildet, welche medialwärts von der hinteren Tröltsch'schen gelegen, lateralwärts das mediane Blatt der letateren, medialwärts den Ambosskörper, nach oben die horizontale Amboss-Pankenwandfalte zur Begrenzung hat. Nach vorn geht sie in die Prussak'sche Tasche uiber, oder, falls eine solche nicht vorhanden ist, erreicht sie ihr Ende am Hammerhals, an welchen sich dann das mediale Blatt der hinteren Tröltsch'schen Tasche inserirt. Nach unten und hinten ist die Tasche offen und mündet in die Paukenhöhle.

Die Falte zwischen Amboss, Hammer und Schuppentheil 
bildet die untere Begrenzang des Hammer-Amboss-Sehuppenraums - unter dieser Bezeichnung wollen wir ihn in der weiteren Betrachtung fiibren. Aber nicht sie allein, sondern auch der Theil der Shrapnell'schen Membran, welcher nach oben und aussen von der Verschmelzung mit jener Falte liegt. Wir werden später sehen, inwiefern diese Thatsache ron Bedeutung ist. Die bisher geschilderten Verhältnisse finden sich nicht immer mit absoluter Regelmässigkeit, sondern lassen mancherlei Varianten zu (Fig. 4).

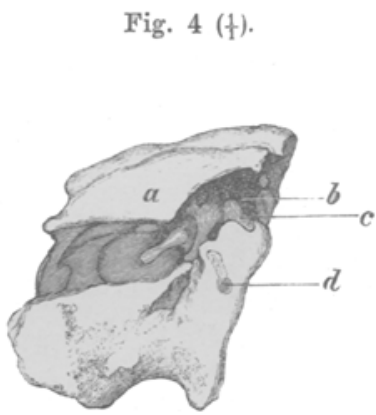

Schnitt wie in Fig. 3.

$a$ Tegmen tympani. $b$ Membran, die Communication zwischen Paukenhöhle und Hanmer-Amboss - Schuppenraum vollständig verschliessend. $c$ Amboss. $d$ vorm derer Semicircularkanal.

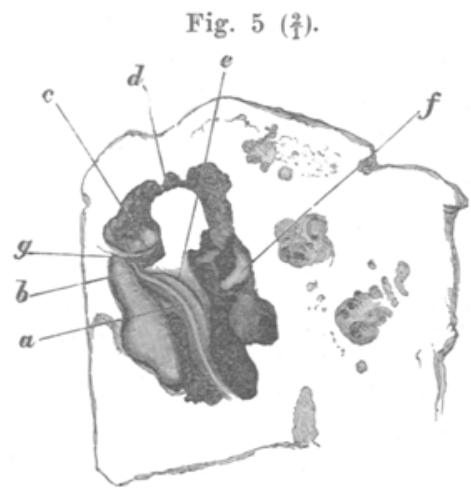

Schnitt dicht hinter dem kurzen Hammerfortsatz senkrecht zur Längsaxe der Pyramide. $a$ Trommelfell. $b$ Membrana flaccida. c Membran zwisohen Hammer, Amboss und Schuppe, von der Membrana flaccida sich abzweigend. $d$ Lig. mallei superius. $e$ Hammer. f Steigbugel. $g$ Prussak'seher Raum.

So findet sich nicht selten, im Kindesalter fast in der Regel, die trapezoidförmige Communicationsöffnung ganz verschlossen dadurch, dass die oben bereits erwähnte Schleimhantfalte zwischen kurzem Ambossschenkel and Paukenwand das Tegmen tympani erreicht und in den freien Rand des Lig. incudis oder mallei superius übergeht. In solchen Fällen würde ein absoluter Abschluss des Hohlraumes von der Paukenhöhle stattfinden.

Die vom Hammer-Amboss zum Schuppentheil hinziehende Falte füllt nicht immer den ganzen Spalt aus, sondern zeigt zuweilen in ihrer hinteren Hälfte Litcken und kann sogar vom Ligamentum externum ${ }^{1}$ ) ab fehlen. In solchen Fällen communicirt

1) Unter Ligamentum externum versteht Helmholtz das Band, welches von der Crista des Hammerhalses ausgehend sich hinter dem Rivinischen Ausschnitt an den Margo tympanicus inserirt. 
die Paukenhöhle mit dem Hohlraum auch durch diesen dreieckigen Spalt.

Ein anderes Mal weicht das horizontale Schleimhautblatt in seiner hinteren Hälfte nach oben $a b$ und inserirt an der oberen Kante des kurzen Ambossfortsatzes. Infolge dessen liegt der vordere lateral vom Hammer gelegene Abschnitt im Vergleich zum hinteren tiefer. Der Boden bildet eine Art Becken, welches eine etwaige Secretansammlung ganz besonders begünstigen dürfte. Bei der vorderen, oberen und den seitlichen Begrenzungen bleiben die Verhältnisse constant.

Betrachten wir nun den Hohlraum als Ganzes, so wïrde seine Gestalt annähernd der einer Birne entsprechen, deren Blï-

Fig. 6 (1).

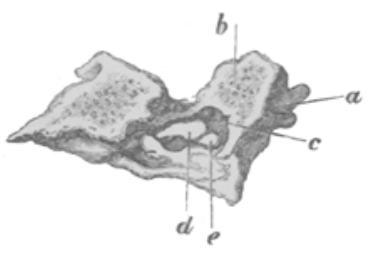

Ansicht des Hammer-AmbossSchuppenraumes von oben. Ein Stiuck hinterer und oberer Gehörgangswand entfernt. a Tegmen tympani. $b$ Sägefläche der hinteren und oberen Gehörgangswand. c Knochenniesche des Schuppentheils. $d$ Amboss. $e$ Hammerkopf.
Fig. $7\left(\frac{2}{1}\right)$.

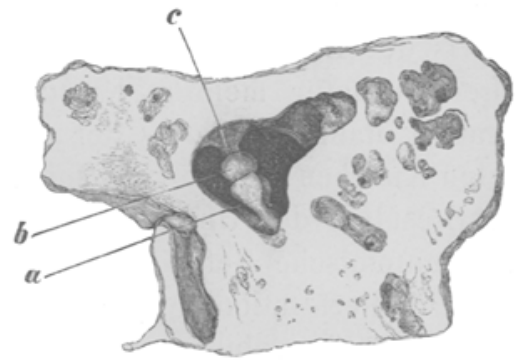

Schnitt senkrecht zur Längsaxe der Pyramide $2 \mathrm{Mm}$. hinter dem binteren Rande des. Trommelfells, vordere Hälfte. $a$ kurzer Ambossschenkel. $b$ Hammerkopf.

c Ligamentum mallei superius.

thenseite nach vorn, Stielseite nach hinten zu gelegen wäre. Die grösste Ausdehnung in frontaler Richtung hat dieser Raum in einer durch den kurzen Hammerfortsatz gelegt gedachten Ebene (Fig. 5). Mittelst der seinen lateralen Abschnitt darstellenden Knochenniesche lagert er sich mehr oder weniger weit über den äusseren Gehörgang. Den Boden bildet das oberste Stiick der Membrana flaccida und die den Spalt zwischen Schuppe, Hammer und Amboss ausfüllende Membran (Fig. 6). Der Hammerkopf und Hals oberbalb der Crista liegt mit seiner lateralen Hälfte im Innern des Raumes. Das Gelenk zwischen Hammer und Amboss befindet sich grösstentheils ausserhalb desselben, nur die vordere Partie der oberen Facette ragt hinein mit dem entsprechenden Stüek des Ambosskörpers und dem kurzen Fortsatze. 
Die Communication mit der Paukenhöhle befindet sich an der medialen Wand des Raumes über dem kurzen Ambossschenkel oder in einzelnen Fällen zwischen dem letzteren und der Schläfenschuppe. Der sich an den Isthmus zwischen Antrum und Paukenhöhle inserirende kurze Ambossschenkel bildet eine Art Septum, wie eine Ansicht des von hinten eröffneten Antrum lehrt (Fig. 7), welches den Aditus in zwei ungleiche Hälften theilt, von denen die laterale, kleinere nach unserem Raume, die mediale, grössere in die Paukenhöhle führt. Hiernach ist der Zusammenhang des Hammer-Amboss-Schuppenraumes mit dem Antrum ein sehr inniger.

Wenn wir so die Existenz eines relativ nicht unbedeutenden, grösstentheils von der Paukenhöhle abgeschlossenen Hohlraumes dargethan haben, so bleibt jetzt zu erweisen, ob sich der Process, welcher zur Perforation des Trommelfells über dem Processus brevis des Hammers führt, auch in jenem Raume abspielt. Es sprechen dafür mehrere Umstände. Einmal die Lage der Perforation, dann die Caries der an der Bildung des Raumes betheiligten Knochen (Margo tympanicus und Gehörknöchelchen, ferner das Resultat der Untersuchung mit der Sonde und schliesslich der anatomische Befund am Präparat.

Die Perforationen haben sehr mannigfaltige Formen. Sie werden häufig gebildet durch Zerstörung der ganzen Membrana Shrapnelli und des Margo tympanicus in grösserer oder geringerer Ausdehnung. Es lassen sich in solchen Fällen der Hammerkopf und Hals, sowie die an denselben aufgetretenen Veränderungen sehen, und der grösste Theil des von den Amerikanern als "Attic" bezeichneten Raumes liegt zu Tage.

Auf der anderen Seite finden sich aber auch Perforationen über dem Processus brevis, die nicht grösser sind als ein Stecknadelstich und hänfig auf der Spitze einer konischen Hervorwölbung der Membrana flaccida sitzen. Bei der Otoskopie kann ein solcher Befund leicht zur Verwechselung mit Polypen Veranlassung geben. Das Erkennen derartig feiner Perforationen wird dadurch noch erschwert, dass hänfig eine Kruste eingetrockneten Eiters sie ganz verdeckt. Sexton (l. c.) beschreibt unter dem Namen "Acute Entzlindung des Attic" das eben dargestellte klinische Bild folgendermaassen: „Die Affection beginnt mit Schmerz, Röthung und Injection der Membrana Shrapnelli; oft wird dieselbe vorgewölbt und kann zu Verwechselung mit einem PolypenAnlass geben." Zwischen den erwähnten Extremen in der Grösse 
der Perforationen findet sich die ganze Reihe der Zwischenformen vertreten. In all den Fällen, in welchen es sich um kleinere Perforationen handelt, ist die Oeffnung fast in unmittelbarer Nähe des Rivinischen Ausschnittes gelegen, mehrere Millimeter entfernt von der Spitze des kurzen Fortsatzes. Wir sahen oben, dass die zwischen den Helmholtz'schen Bändern anterius und externum ausgespannte Membran, welche mit der Prussak'schen oberen Begrenzungsmembran identisch ist und einen Theil der von uns geschilderten Hammer-Amboss-Schuppenfalte darstellt, in der Regel nicht am Rivinischen Ausschnitt entspringt, wie Prussak meint, sondern weiter unterhalb von der Membrana flaccida sich abzweigt. Wenn nun die Eitersenkung entlang dieser Membran erfolgt, so muss auch der Durchbruch nach anssen in der Nähe des Rivinischen Ausschnittes stattfinden. Gerade der Sitz der Perforationen an dieser Stelle spricht gegen die Annahme Morpurgo's und Hessler's, dass die Eiteransammlung in der Prussak'schen Tasche zu Stande kommt. Wäre dies der Fall, so mïsste der Durchbruch dicht itber dem Processus brevis erfolgen, da die obere Fläche des kurzen Fortsatzes die tiefste Partie des Prussak'schen Raumes darstellt. Allerdings brechen ja auch in der Membrana tympani die Exsudate nicht immer an der tiefgelegensten Stelle durch, aber in der überwiegenden Mebrzahl finden sich doch die Perforationen im unteren, vorderen Quadranten. Die gleiche Beobachtung bezïglich der Durchbruchsstelle der im Hammer-Amboss-Schuppenraum befindlichen Exsudate hat auch Sexton gemacht. Er sagt (1. c.): „In dem eingesunkenen Sinus der Membrana Shrapnelli findet sich eingedickter Eiter, Epithel, Polypen. Der Durchbruch erfolgt durch die Membran oder das äusserste Ende der Gehörgangsplatte."

Das Politzer'sche Höhlensystem hat freilich seinen Sitz am Rivinischen Ausschnitt. Es ist aber ein verhältnissmässig so kleines und zartes Gebilde, dass es wohl für die vorliegende Frage nicht in Betracht kommen kann.

Bei lange bestehender Eiterung kommt es gewöhnlich zu cariöser Schmelzung des Rivinischen Ausschnittes, welche sich mehr oder weniger weit in den Gehörgang hinein erstrecken kann und auch zuweilen die benachbarten Theile des Margo tympanicus betrifft. Sehr oft ist dieses Ereigniss durch die Ocularinspection zu constatiren. Das häufige Vorkommen von polypösen Wucherungen an jener Stelle, der Fötor, welcher gegen die gewöhnlichen Behandlungsmethoden sich äusserst hartnäckig 
erweist, müssen ebenfalls den Verdacht anf Caries lenken, selbst wenn das Aussehen der Perforation nicht dafür zu sprechen scheint. Zur Illustration dafür diene der bei der Behandlung durch Hammerextraction geschilderte Fall Minna O. (Fall 16). Während dem otoskopisehen Befunde nach eine Caries des Grehörgangsrandes nicht vorzuliegen schien, ergab das nachträgliche Evidément mit dem scharfen Löffel, dass bereits eine sehr bedeutende Zerstörung stattgefunden hatte. In zwei Fällen konnten wir einen Durchbruch bei intacter Membrana flaccida, vor derselben im Gehörgang gelegen, beobachten. Der Eiter hatte sich hier durch den Boden der dem Schuppentheil des Schläfenbeines angehörenden Knochenniesche einen Ausweg verschafft.

In gleicher Weise wie den Margo tympanicus betrifft die Caries die Gehörknöchelchen, vorzüglich den Hammer. Dieses Ereigniss ist so häufig, dass $\mathrm{Seh}$ wartze schon in seiner „Pathologischen Anatomie des Ohres" (1878) S. 88 und später in seinem "Lehrbuch der chirurgisehen Krankheiten des Obres" (1884) S. 287 das Vorhandensein ,circumscripter Granulationen am oberen Pole des Trommelfells in der Umgebang des Processus brevis" und einer Fistelöffnung iiber dem kurzen Hammerfortsatz als ein bei der Diagnose von Caries des Hammerkopfs zu verwerthendes Zeichen anfuhrt. Die Thatsache wird auch von Burnett ${ }^{1}$ ), Bezold ${ }^{2}$ ), Politzer ${ }^{3}$ ) vollinhaltlich bestätigt.

Fig. $8\left(\frac{2}{1}\right)$.
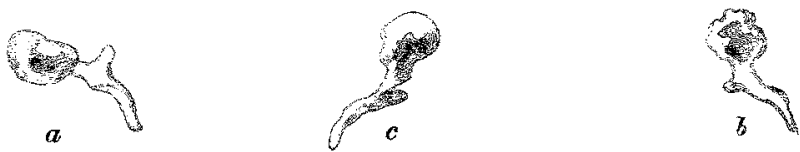

Hämmer mit cariösen Defecten.

$a$ beginnender, $b$ fortgeschrittener, $c$ hochgradiger Defect.

Bei der Durchsicht einer grösseren Anzahl cariöser Hämmer der klinischen Sammlung in Halle konnte constatirt werden, dass bei geringer Zerstörung immer die Stelle über

1) Treatise on the Ear. 1877. p. 336 . American Journal of Otol. $\nabla, 3$. No.1. 1881 .

2) Dieses Archiv. Bd. XV. S. 1 ff.

3) Lehrbuch. 1882. S. 485. 
der Crista des Hammerhalses zuerst betroffen war, bei grösserer Ausdehnung des Processes wurde der Hammerkopf immer weiter excavirt gefunden (Fig. 8). Am längsten bleibt der Theil des Knochens erhalten, welcher die Gelenkfläche trägt.

Da die den Raum unten abschliessende Begrenzungsmembran an der Crista des Hammerkopfes inserirt, so wird das von dem auf ihr lastenden Secrete ausgehende Zerstörungswerk am Hammer dicht oberhalb der Crista beginnen miussen. Die Caries breitet sich dann weiter in dem innerhalb des Raumes gelegenen Theile des Hammers aus und verschont am längsten die ausserhalb gelegenen, d. i. die Gelenkfläche tragenden Partien. An einigen Ambossen (Figur 9) konnte gleichfalls an der lateralen Seite des Körpers und kurzen Fortsatzes, also an der dem Raum zugekehrten Fläche eine cariöse Zerstörung constatirt werden. Würde Fig. $9\left(\begin{array}{l}2 \\ 1\end{array}\right)$.

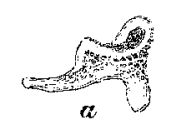

Cariöse Ambosse.

$a$ beginnende, $b$ fortgeschrittene Zerstörung. sich nun der Process in dem

Prussak'schen Raume abspielen, so müsste nothwendigerweise doch der Hammerhals unterhalb der Crista und der kurze Fortsatz mit Vorliebe der Sitz cariöser Erkrankungen sein, und es miissten im Beginn sich die ersten Spuren an diesen Theilen zeigen. Ein derartiges Vorkommen war jedoch an keinem der Präparate zu constatiren.

Die Resultate der Sondenuntersuchung führen ebenfalls den Beweis, dass die Eiterung in dem von uns beschriebenen Raume stattfindet. Die Lage und Ausdehnung der hinter der Perforation gelegenen Höhle macht eine Identität mit unserem Hohlraum zur Gewissheit. Hessler ${ }^{1}$ ) sagt darüber: „Die cariöse Höhle liegt nun nach den Ergebnissen der Sondirung nicht nach vorn und innen, sondern nach innen hinten und oben aussen von ihr (der Perforation). Man muss, um in diese Region zu kommen, den Knopf der Sonde mehr oder weniger lang und winklig umbiegen." Die Ergebnisse unserer Sondirungen stimmen mit denen Hessler's völlig überein und liefern den Beweis, dass der „cariöse" Raum über dem Gehörgang liegt.

In einem Falle konnte ich eine Eiteransammlung in dem von der Paukenhöhle vollständig abgeschlossenen Raume durch

1) 1. c. S. 140 . 
Section constatiren. Es betraf derselbe ein 6 Monate altes, an Tuberculose gestorbenes Kind. Das linke Felsenbein, welches mir zur Verfügung stand, wurde durch einen senkrecht zur Längsaxe der Pyramide $2 \mathrm{Mm}$. hinter der hinteren Insertion des Trommelfells angelegten Sägeschnitt getheilt, dann durch einen senkreeht dazu durch die Paukenhöhle in der Nähe der Labyrinthwand verlaufenden zweiten Schnitt die mediale Fläche des Trommelfells mit Hammer und Amboss und grossem Theile des Tegmen freigelegt. Die Paukenhöhle enthielt Eiter. Die Paukenschleimhaut war stark verdickt, ebenso die von der oberen Kante des kurzen Ambossschenkels, Hammerkopfes etc. nach dem Tegmen und der Schläfenschuppe zu aufsteigende Membran, welche einen totalen Abschluss nach oben herbeiführte. Nach Trennung dieser Membran findet sich zwischen Hammer-Amboss einer- und Schläfenwand andererseits eingedickter Eiter, nach dessen Entfernung die untere Begrenzungsmembran vollständig, aber ebenfalls stark verdickt, zu Tage tritt. Die von aussen am Rivinisehen Ausschnitt angedrickte Sonde schimmert, sobald das Präparat gegen das Licht gehalten wird, an der unteren Begrenzungsmembran deutlich durch. Hier war also ein völliger Abschluss zu constatiren, vielleicht erst zu Stande gebracht durch die pathologischen Vorgänge in der Paukenhöhle.

Die grösste Anzahl von Fällen mit „Perforationen über dem Processus brevis" ist chroniseher Natur. Unter 30 Kranken fanden sich nur 2 mit acuter Affection. Dennoch aber wird wohl grösstentheils die chronische Form den Ausgang von einem acuten Anfangsstadium genommen haben, gerade so, wie es auch bei den meisten anderen chronischen Paukenhöhleneiterungen der Fall zu sein pflegt. $O b$ nun die Erkrankung isolirt in dem Raum sich abspielt, oder ob sie im Anschluss an eine allgemeine Entzündung der Paukenhöhle erfolgt, lässt sich nachträglich nicht entscheiden. Sexton (1. c.) ist der Ansicht, dass eine isolirte Entzündung eintreten kann, wenn er sagt: „Eine Entzündung des, Attic ${ }^{`}$ kann obne Betheiligung der iibrigen Paukenhöble entste! en; sie beginnt nach acuten Exanthemen oder nach Anwendung der Nasendouche infolge Eindringens von Flüssigkeit durch die Tuba mit Sebmerz und Röthung der Membrana flaccida, Gefässinjection am Gehörgang, Austritt von blutigem Serum. Oft ist Periostitis der Nachbarschaft dabei." Ich habe eine derartige isolirte Erkrankung nicht beobachtet, wohl aber ein Mitbefallenwerden bei allgemeiner Entzïndung der Paukenhöhle. 
Bei einem Kinde von 4 Jahren war ohne nachweisbare Ursache eine doppelseitige Entzündung der Paukenhöhle mit Exsudatbildung eingetreten. Nach 8 tägigem Bestehen zeigte sich bei der Untersuchung das Trommelfell rechts stark vorgewölbt, injicirt und auf der Höhe der Wölbung eine ganz feine Perforation. Das linke Trommelfell war ebenfalls stark vorgewölbt und injicirt, doch ohne Perforation. Die beiderseitig vorgenommene Paracentese entleerte massenhaftes, grösstentheils schleimiges Exsudat. Tags darauf war das rechte Trommelfell in toto abgeflacht, links dagegen nur mit Ausnahme der Membrana Shrapnelli.. Diese wölbte sich noch sackartig in den Gehörgang. Am folgenden Tage war der Befund derselbe und es sollte am nächsten die Paracentese der Shrapnellmembran vorgenommen werden. Dies wurde aber nicht mehr nöthig, da die Vorwölbung an diesem Tage spontan zurückgegangen war.

Es war also in diesem Falle jedenfalls zur Entzündung des Raumes über der Membrana Shrapnelli gekommen, gleichzeitig mit Entzïndung der ïbrigen Paukenhöhle. Durch Schwellung der Schleimhaut wurde die Communication mit der Paukenhöhle verlegt.. Daher konnte das Secret des oberen Raumes nach der Paracentese nicht sofort abfliessen. Die Entleerung trat erst ein, nachdem durch Entlastung der übrigen Paukenhöhle vom Secretdruck eine Abschwellung der Schleimhaut und ein Wiederwegsamwerden der Communicationsöffnung erfolgte.

Morpurgo ${ }^{1}$ ) beschreibt einen Versuch, der unsere Ansicht ïber das Zustandekommen des Processes bestätigt. In einer Reihe vun Fällen mit einfachem chronischen Mittelohrkatarrh durchstach er die keine Abnormitäten zeigende Membrana flaccida und konnte bei darauf folgender Lufteintreibung das Durchdringen der Luft constatiren $(14 \mathrm{mal})$. In einem Falle kam es zu einer ziemlich intensiven Hyperämie im Bereiche der Membrana flaccida. Die Wunde heilte aber trotzdem in 2 Tagen. Nach einer am 5. Táge an derselben Stelle wiederholten Punction drang keine Luft mehr durch. Die einige Zeit später nach Ablauf der Hyperämie zum dritten Male vorgenommene Punction führte wieder zu einem Perforationsgeräusch. Es war also hiernach durch die sich an die erste Paracentese anschliessende Entziindung die Communication mit der Paukenhöhle aufgehoben worden und trat erst wieder ein nach Ablauf des entzuindlichen Processes.

1) 1. c. S. 271. 
Wenn nun nicht durch eine geeignete Behandlung. für $\mathrm{Ab}$ fluss des Secretes Sorge getragen wird, so kann es bei längerem Bestande der Schleimhautschwellung zur Verwachsung der sich beriihrenden Theile und dadurch zu danerndem Abschluss der Communicationsöffnung kommen. In diesem Falle verlaufen die Processe in den beiden Höhlen unabbängig von einander. Der Paukenhöhlenraum bietet aber für eine spätere Heilung noch guinstigere Verhältnisse als der obere Raum, da durch die in ihn mündende Tube ein Secretabfluss ermöglicht wird. Daher mag es auch kommen, dass in der grössten Anzahl der chronischen Fälle mit Perforation über dem kleinen Fortsatz die Paukenhöhle frei von Erkrankungen ist. Nichtsdestoweniger bleibt aber noch eine ganze Anzahl solcher Fälle iubrig, bei welchen die Affection der Paukenhöhle keinen so günstigen Ausgang genommen, sondern zu ähnlichen Folgen geführt hat, wie im oberen Raum. Das lehren uns die Trommelfellbefunde, bei welchen sich neben der Perforation über dem kurzen Fortsatz noch eine zweite im Trommelfell findet. Burnett (Lehrbueb) stellt das Vorkommen einer derartigen doppelten Perforation als ein besonders seltenes Ereigniss hin. Wir konnten unter 33 Fällen diese Thatsache $8 \mathrm{mal}$ constatiren, bei 3 Fällen fehlte das ganze Trommelfell, nur zu beiden Seiten des Hammers zogen Stränge, welche denselben in seiner Lage hielten, nach dem Margo tympanieus hin.

Wenn nun eine Eiteransammlung in dem Hammer-AmbossSchuppenraum stattgefunden hat, so wird bei mangelnder Entleerung infolge der bei Mittelohreiterungen nie fehlenden Erreger eine Zersetzung des Secretes erfolgen. Dasselbe kann alsdann seine zerstörende Thätigkeit auf die benachbarten Knochen ausiuben und zu Caries des Hammers, Ambosses, sowie der Partie des Schuppentheils, welche den Raum lateral begrenzt, führen. Kommt es zum Durchbruch des Eiters nach aussen, so wird derselbe naturgemäss an der tiefsten Stelle stattfinden, also in der Membrana Shrapnelli, dort, wo sich die untere Begrenzungsmembran von ihr abzweigt. Bei cariösen Processen kommt es in der Regel zur Bildung von Granulationen, und es finden sich deshalb dieselben so sehr häufig ( $16 \mathrm{mal}$ bei 30 Fällen, nach Bezold ${ }^{1}$ ) 4 mal unter 7 Fällen) bei dieser Form der Eiterung, gewöhnlich die Perforation ausfüllend, so dass bei der Untersuchung die

1) Dieses Arch. Bd. XV. S. 18. 
Oeffnung vollständig verdeckt ist und erst nach Entfernung der Wucherungen zu Tage tritt. Man hat sich hierbei zu hüten vor der Verwechselung mit der oben erwähnten zapfenförmigen Vorwölbung der Schrapnellmembran, veranlasst durch angehäuftes Secret.

Da durch die Abgeschlossenheit des Raumes die Stagnation und Eindickung eitrigen Inhalts begünstigt wird, so ist es erklärlich, dass so häufig Cholesteatombildung eintritt (10 mal bei 30 Fällen, nach Bezold 3 mal unter 7 Fällen). Ueber die Entstehung des Cholesteatoms im Gehörorgan spricht sich v. Tröltsch ${ }^{1}$ ) folgendermaassen aus: „Nachdem die eingetrocknete Eitermasse eine gewisse Härte und Grösse erreicht hat, wirkt sie allseitig als Reiz auf die Umgebung und deren Auskleidung, deren zellige Producte selbst wieder zur Vergrösserung der ursprünglichen Masse und somit der Druckreizung beitragen, auch unter diesen pathologischen Verhältnissen nicht blos in besonderer Menge, sondern auch von veränderter Gestalt und Art geliefert werden, so dass sie geschichteter Epidermis gleichen und als Producte einer desquamativen Entzündung der Wandauskleidung figuriren können."

Es ist nun aber zum Zustandekommen der Folgezustände einer Eiterung im oberen Raum nicht nöthig, dass ein vollständiger Abschluss von der Paukenhöhle eintritt. Die buchtenartige Form des Raumes ermöglicht auch ohnedem das isolirte Fortbestehen einer ursprünglich in der ganzen Paukenhöhle sich abspielenden Entzündung, wie wir ein solches in analoger Weise auch im Antrum mastoideum nicht selten finden. Für das Fehlen eines Abschlusses gegen die Paukenhöhle spricht das Vorhandensein des Perforationsgeräusches, welches Morpurgo in 25 Proc. seiner Fälle fand. Wir konnten unter 15 Fällen 6 mal ein Fehlen, 4 mal ein Vorhandensein und 5 mal den späteren Eintritt des ursprünglich fehlenden Perforationsgeräusches constatiren. Ein Fehlen dieses Geräusches setzt nicht immer zugleich Fehlen einer Communication mit dem übrigen Paukenhöhlenraum voraus. Es kann vielmehr diese Verbindung durch eingedicktes Secret oder durch Granulationsbildung verlegt sein. Gar nicht selten lässt sich die Bemerkung machen, dass bei anfangs fehlendem Perforationsgeräusch infolge einer Lufteintreibung in der zuerst freien Oeffnung eine Granulation erscheint, nach deren

1) 1. c. S. 495 . 
Beseitigung die Luft durchdringt, oder dass dies Ereigniss eintritt nach einer Ausspülung des Raumes mit der gebogenen Canüle und nach Entfernung eingedickten Eiters. Andererseits kann es aber auch zu Secretaustritt nach der Luftdouche kommen, oḥne dass ein Perforationsgeräusch eintritt. Wahrscheinlich wirkt dann die in die Paukenhöhle getriebene Luft comprimirend auf die membranösen Theile des oberen Raumes und führt so zu einer theilweisen Entleerung desselben.

Die Stagnation des sich zersetzenden Secretes und die in der Regel bestehende Caries bringen es mit sich, dass diese Formen der Eiterung stets mit sehr erheblichem Fötor verbunden sind. Die Neigung zur Bildung vón Granulationen fübrt häufig zu einem Hineinwachsen derselben in die Perforation und bedingt dadurch einen verminderten Abfluss der Entzündungsproducte. Infolge dessen ist die Gefahr einer Ausbreitung des Krankheitsprocesses auf das so äusserst nahe Tegmen mit ihren schweren Folgen eine sehr grosse. Bezold ${ }^{1}$ ) macht darauf aufmerksam, dass die mit Perforation der Membrana flaccida einhergehenden Eiterungen unter den chronischen relativ am häufigsten direct zum Tode führen. Sexton (l. c.) erwähnt, dass bei Kindern ein Uebergehen der Entzïndung auf die Schädelhöhle wegen des Offenstehens der Sutura petrosquamosa besonders zu fürchten sei.

Die Nähe am Tegmen tympani mag auch die so bäufig bei dieser Form der Eiterung auftretenden Symptome, Kopfschmerz, Schwere im Kopf und Schwindel, erklären. Politzer führt die Ursache dieser Symptome in einzelnen Fällen auf eine abnorme Steigerung des Labyrinthdruckes, v. Tröltsch auf eine von den Kंnochenwänden des Mittelohrs auf die harte Hirnhaut und Hirnvenen fortgepflanzte Hyperämie zurïck.

Die meisten Fälle von Perforation über dem Processus brevis werden ihre Entstehung dem Kindesaiter verdanken. Die Aussagen der Patienten über den Zeitpunkt des Beginns ihres Leidens sind gewöhnlich ungenau. Sie pflegen den Anfang erst von dem Moment ab zu datiren, wo sie erheblichere Beschwerden gefühlt haben. Wie häufig kommt es vor, ganz besonders gerade bei diesen Formen, dass sich auf dem von den Patienten für gesund gehaltenen Ohre Residuen von früheren Eiterungen finden, dass aber die jemalige Existenz einer Erkrankung dieses Ohres von dem Träger in Ab-

1) Dieses Arch. Bd. XXI. S. 231. 
rede gestellt wird. Es konnte diese Thatsache unter 30 Fällen 5 mal constatirt werden. Der Ansicht, dass die Eiterungen schon in frühem Lebensalter entständen, ist auch Hessler ${ }^{1}$ ), wenn er sagt: „Statistische Nachweisungen datiren den Beginn solcher Eiterungen schon häufig in die früheste Kindheit zurück, und welcher Grund soll vorliegen, anzunehmen, dass der anatomische Befund im $\mathrm{Ohr}$ sich erst allmählich verändert und schliesslich zur Perforation der Shrapnell'schen Membran gefuhrt hat, wie wir sie viele Jahre nach Beginn der Affection finden, wenn sich die Patienten, denen nun die sich gegen Otorrhoen, wenn sie sebmerzlos sind, gleichgültig verhaltenden Eltern nicht mehr zur Seite stehen, den Ohrenärzten vorstellẹn und wegen der fötiden Eiterung Heilung suchen?" Auch nach Morpurgo ${ }^{2}$ ) entstehen die meisten Formen im Kindesalter.

Dass bei der Erkrankung des Hammer-Amboss-Schuppenraums, welche so häufig auch die Gehörknöchelchen befällt, eine Störung des Hörvermögens eintreten wird, kann nicht wunderbar erscheinen. Diese Störung ist aber durchaus nicht proportional den objectiven Befunden. Bei grossen Defecten ist die Perception für Flüstersprache oft erstaunlich gut, während sie bei anscheinend geringfügigen Veränderungen ganz erheblich herabgesetzt ist. So konnte ich bei einer Frau, welche an einer doppelseitigen Eiterung des oberen Raumes litt, mit Zerstörung eines grossen Theils der oberen Gehörgangswand und frei $\mathrm{zn}$ Tage liegendem Hammerkopfe ein Hörvermögen für Flüsterzahlen auf dem einen $\mathrm{Ohr}$ auf 5, auf dem anderen auf 4 Meter feststellen, nachdem die Eiterung geheilt war.

Die Resultate von 23 Hörprüfungen auf Flüsterzahlen sind folgende:

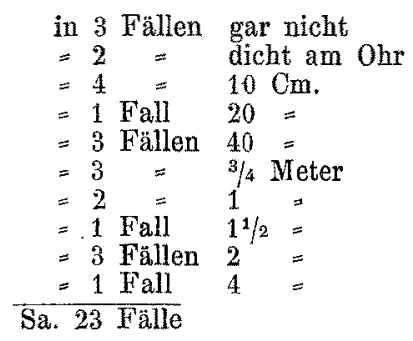

Ebensowenig wie der objective Befund steht die Dauer der Erkrankung im Verhältniss zu der Gehörsstörung. Soviel lässt 
sich jedenfalls sagen, dass die Hörverminderung in vielen Fällen nicht ausschliesslich durch die Störung im mechanischen Apparat bedingt ist.

Nicht ganz selten sind die chronischen Eiterungen, welche zur Perforation uiber dem Processus brevis führen, complicirt mit Erkrankungen des Antrum mastoideum (Bezold, Politzer, Hessler, Morpurgo l. c.). Ob die Erkrankung im Hammer-AmbossSchuppenraum oder die im Antrum die primäre, bezugsweise secundäre ist, oder ob beide aus einer Grundursache, allgemeiner Entzündung der Paukenhöhle und Nebenräume entstanden sind, lässt sich ex post nicht entscheiden. Jedenfalls ist bei der anatomischen Anordnung der beiden Räume (s. oben) der Uebergang des Erkrankangsprocesses von dem einen in den anderen nicht anffallend. Die Uebertragung erfolgt wahrscheinlich durch Vermittelung des kurzen Ambossschenkels oder des Margo tympanicus, der ja mit seinem hinteren Abschnitt ziemlich nahe an den Aditus ad antrum heranreicht. In der That finden sich bei der Caries der Warzenfortsatzhöhle sehr hänfig am hinteren oberen Trommelfellrand durchwachsende Granulationen, als Zeichen, dass die demselben benachbarte Knochenpartie erkrankt ist. Dass der cariöse Process sich weiter von dort her ausbreiten und sich in den nieschenförmigen, über dem Gehörgang liegenden Raum der Schläfenschuppe erstrecken kann, scheint sehr natürlich.

Was die Fortpflanzung durch den kurzen Ambossschenkel anbelangt, so liess sich an einigen Knöchelehen der klinischen Sammlung in Halle eine cariöse Zerstörung seines hinteren Endes constatiren, so dass die Annahme berechtigt ist, Fig. $10\left(\frac{2}{1}\right)$. die Erkrankung habe sich vom Rande des Aditus

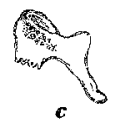

Cariöse Einsehmelzung des kurzen Ambossschenkels. ad antrum, wo der Amboss gelenkig inserirt, auf den kurzen Fortsatz fortgesetzt (Fig. 10). In einem Falle von acuter Caries des Warzenfortsatzes bei einem Kinde wurde bei der Ausräumung der Warzenfortsatzhöhle auch der Amboss mit entfernt. Der kurze Schenkel desselben war grösstentheils cariös eingeschmolzen.

So ungünstig auch die Chancen für eine Spontanheilung der in Rede stehenden Eiterung sind, so kommt doch eine solche zu Stande. Wir konnten unter 1000 Ohrenkranken diese Thatsache 2 mal, 1 mal davon doppelseitig constatiren. Der seinerzeit weit in die obere Gehörgangswand vorgedrungene Defect war dureh eine feine Narbe geschlossen. Die betreffenden Patienten wussten 
von einer früheren Eiterung nichts. In den meisten Fällen tritt jedoch die Spontanheilung nieht ein, und es bleibt deshalb Aufgabe der Therapie, zu versuchen, ein gleich gïnstiges Resultat herbeizufiihren.

Die therapentischen Vorschläge Morpurg 0's') bestehen in intratympanalen, desinfieirenden Einspritzungen, kaustischer Behandlung, blutiger Erweiterung der Perforation, Alkoholeinträufelung. Der ersteren Methode, welche oft massephaftes Secret entfernt, ribmt er nach eine baldige Verminderung des Geruches und der Secretion; eine Heilung soll aber nicht eintreten. Die blutige Erweiterung ist erforderlich bei enger Perforationsöffnung; er empfiehlt, die Dilatation nach oben vorzunehmen, um eine Eröffnung der Pauke und eine Infection derselben zu vermeiden. Gewöhnlich ist aber die obere Begrenzung knöchern, und es wird in den seltensten Fällen eine Dilatation in dieser Richtung gelingen. Die Erweiterung wird besser durch Aetzmittel oder durch Galvanokaustik erreicht. Keine zufriedenstellenden Erfolge sah Morpurgo bei der Anwendung kanstischer Lösungen. Am besten wirkte bei ihm die Einträufelung von Alkohol combinirt mit tympanalen Ausspülungen.

Jedenfalls ist bei der Alkoholbehandlung der vorliegenden Formen von Eiterung wegen der unmittelbaren Nachbarschaft des Schädelraumes grosse Vorsicht erforderlich. Schwartze ${ }^{2}$ ) erwähnt 2 Fälle, wo bei Patienten, welche mit Alkoholeinträufelungen behandelt waren, der Tod unter den Erscheinungen der Pyämie und Meningitis eingetreten war. Die Ursache ist wahrseheinlich in einer durch den Alkohol bewirkten Thrombenbildung mit nachberiger Verjauchung zu suchen.

Hessler (l. c.) hat gute Resultate erreicht dadurch, dass er durch die Perforation die der Sondenuntersuchung entsprechend nach innen hinten und oben aussen von ihr liegende Hohle möglichst allseitig mit Lapis in Substanz betupfte. Zwar kamen danach nicht ganz selten entzindliche Verschwellungen in der Umgebung der Perforation mit geringerer oder grösserer Reaction vor, jedoch wichen dieselben regelmässig der Anwendung von Priessnitz'schen Umschlägen. Zur Erweiterung zu enger Perforationen benutzte er mit gutem - Erfolg den Galvanokauter. Eine Vereinigung des oberen Raumes mit der Paukenhöhle zum

1) 1. c. S. 275 .

2) Lehrbuch. S. 204. 
Zweck von Durchspïlungen von der Tuba aus durch einen Schnitt, welcher von der Perforation in der Membrana flaccida entlang der hinteren Kante des Hammergriffes verlief́, währte nur wenige Tage. Nach der Verheilung der Schnittwunde war auch die Communication wieder aufgehoben.

Bei der Behandlung kommt es darauf an, zu unterscheiden zwischen den Fällen, welche, wie das fehlende Perforationsgeräusch andeutet, von der übrigen Paukenhöhle abgeschlossen sind, und denjenigen, welche mit der Paukenböhle communiciren. In letzterem Falle werden, vorausgesetzt, dass die Perforation weit genug ist, wie ja die Ocularinspection zeigt, und die Communicationsöffnung mit der Paukenhöhle hinreichende Grösse hat, was durch Auscultation festzustellen sein wird; in diesem Falle werden Massendurchspïlungen von reiner Kochsalzlösung ( $3 / 4$ Proc.), wie sie Schwartze (l. c.) empfiehlt, mit Vortheil angewendet. Schwartze (l. c.) schlägt vor, bei den hochgelegenen Perforationen behufs leichterer Durchspülung der Paukenhöhle eine Gegenöffnung im Trommelfell anzulegen. Allerdings wird dadurch die Durchspülung der Paukenhöhle leichter werden, aber der Wasserstrom wird eben diesen leichteren Weg vorziehen und den oberen Raum, selbst wenn Communication desselben mit der Paukenhöhle besteht, nur wenig oder gar nicht berühren. Gerade das Bestehen nur einer Perforation zwingt das Wasser, seinen Weg durch diese zu nehmen und ermöglicht so eine energische Bespülung dèr erkrankten Theile. Wir konnten bei Fällen von doppelter Perforation fast stets die Beobachtung machen, dass nach Durchspülungen von der Tuba aus der obere Raum kaum beeinflusst wurde. Etwas Anderes ist es, wenn auch die Paukenhöhle Secret enthält; dann ist die Paracentese zur Heilung dieser Affection geboten; nicht aber, um einen Einfluss auf die Erkrankung des oberen Raumes auszuüben.

Mittelst consequenter Massendurehspülungen per tubam wurde die Heilung folgender Fälle ${ }^{1}$ ) erreicht.

1. Adolf P., 13 Jahre. Das linke $\mathrm{Ohr}$ hat seit der Kindheit geeitert und häufig stark gerochen, Schmerzen und Kopferscheinungen fehlten. Rechts Residuen chronischer Eiterung. Links Verkalkung im

1) Die im Folgenden aufgeführten Krankengeschichten betreffen sämmtlich Patienten, welche in der kgl. Universitäts-Ohrenklinik zu Halle.a/S. behandelt wurden. Für die gütige Ueberlassung des Materials bin ich dem Director der Klinik, Herrn Geh. Rath Schwartze, zu grossem Danke verpflichtet. 
Trommelfell; vorn oben über dem Processus brevis hängt eine polypöse Wucherung herab. Perforationsgeräusch nicht hörbar. Der Polyp wird mit der Schlinge abgetragen, der Rest mit Lapis und Chromsäure geätzt. 2 Tage später ist ein breites Perforationsgeräusch hörbar. Es zeigt sich in unmittelbarster Nähe des Rivinischen Ausschnittes eine beinahe hanfkorngrosse Perforation. Durchspülungen mit Salzwasser entleeren Schleimeiter. Flüsterzahlen 1 Meter. Die Irrigationen per tubam werden täglich wiederholt. Der Geruch nach 4 Tagen aufgehoben. Eiterung nach 4 Monaten beseitigt. 3 Wochen später ist an Stelle der Perforation eine feine, etwas eingezogene Narbe zu sehen. Das Hörvermögen ist bis auf 4 Meter für Flüstersprache gestiegen.

2. Otto J., 11 Jahre, ist seit Jahren schwerhörig. Links ein von vorn oben ausgehender Polyp. Nach dessen Entfernung tritt eine Perforation in der Membrana Shrapnelli zu Tage. Breites Perforationsgeräusch, Fötor, Durchspülungen per tubam. Der Rivinische Ausschnitt cariös erweicht. Nach einigen Tagen Verschwinden des Fötor. Nach 10 Monaten hat die Eiterung anfgehört. Man sieht durch die Perforation in die von einer epidermisähnlichen Haut ausgekleidete trockene Höhle.

Für die Fälle, welche nicht mit der Paukenhöhle communiciren, giebt es zwei Wege, auf welchen therapeutisch vorgegangen werden kann, erstens vom Gehörgang her durch die Perforation und zweitens von der Tuba aus nach Herstellung einer ausgiebigen und bleibenden Communication mit der Paukenhöhle. Sind Polypen vorhanden, so ist es nothwendig, erst diese zu entfernen, einmal aus therapeutischer Rücksicht, dann aber, weil so uiberhaupt erst das Vorhandensein oder Fehlen einer Communication mit der Paukenhöhle diagnosticirt werden kann. Ein die Perforation ausfüllender Polyp kann das Zustandekommen des Perforationsgeräusches hindern und so einen Abschluss des Hohlraums gegen die Paukenhöhle vortäuschen. Grössere Polypen lassen sich mit der Wilde'schen Schlinge abtragen, kleinere werden mit Lapis in Substanz oder mit Chromsäure, welche sich ebenso wie jener an eine Sonde anschmelzen lässt, oder durch Galvanokaustik zum Schwinden gebracht. Liegt jetzt die Perforation frei zu Tage, so lassen sich durch Einführung der von Schwartze angegebenen Antrumröhrchen ${ }^{1}$ ) in die Höhle desinficirende Ausspülungen derselben obne Schwierigkeit vornehmen. Wir benutzten in der Halle'schen Ohrenklinik als Spülflüssig-

1) Zuerst beschrieben und abgebildet in diesem Archiv. Bd. XIV. S. 225, später auch im. Lehrbuch der chirurg. Krankheiten des Ohres. S. 324. 
keiten gewöhnlich 1-2 proc. Carbolwasser oder Sublimatlösnng 1:2000-5000. Die Einführung des Röhrchens ist bei einiger Uebung und etwas Vorsicht nicht schmerzhaft, jedenfalls nicht schmerzhafter als eine Sondirung. Infolge einer solchen Ausspïlung entleeren sich oft grosse Mengen eingedickten Secretes von gewöhnlich penetrantem Geruch, sowie Cholesteatommassen, auf deren hänfiges Vorkommen bei der vorliegenden Krankheitsform oben bereits hingewiesen wurde. Nach einigen derartigen Ausspülungen pflegen die Erscheinungen von Kopfschmerz, Eingenommensein, Schwindel in der Regel zu verschwinden. Irrigationen in den Gehörgang können die Anwendung des Antrumröhrchens in keiner Weise ersetzen. Wir konnten uns wiederholt ubberzengen, dass nach wochẻnlangen Ausspülungen des Gebörgangs die einmalige Application des Röhrchens noch grosse Mengen bröckligen Inhalts zu Tage förderte. Dass es entgegen Morpurgo's Annahme bei consequenter Anwendung dieses Verfahrens zur Ausheilung der Eiterung kommen kann; lehren die folgenden Krankengeschichten.

3. Hermann H., 14 Jahre, weiss nieht, wie lange er ohrenleidend ist. Rechts über dem Processus brevis befindet sich eine weit in die obere Gehörgangswand ragende pfefferkorngrosse Perforationsöffnung, welche in einen allseitig begrenzten Hohlraum führt. In demselben liegt aufgeschichtet dickes, käsiges Secret. Die Massen werden zuerst mit dem Löffel, soweit sie erreichbar sind, entfernt, alsdann mit dem Antrumröhrehen ausgespült. Der Inhalt erweist sich als krilmliger Detritus von ungeheurem Fötor. Die Paukenhöhle ist frei, Perforationsgeräuseh fehlt und ist nie eingetreten. Unter täglicher Anwendung des Röhrchens bleibt der Fötor nach 4 Tagen weg, die Kopfschmerzen, an dénen der Knabe litt, verschwanden. Nach $11 / 4$ Jahr hat die Eiterung aufgehört. Heute $1 \mathrm{Jahr}$, nachdem der Kranke aus der Behandlung entlassen ist, findet sich die Höhle ganz trocken. Die Perforation ist persistent geblieben. Flüstern 5 Meter.

4. Emma H., 22 Jahre, leidet infolge von Scarlatina seit dem 4. Lebensjahre an doppelseitiger Eiterung. Rechts grosse cariöse Höhle in den Warzenfortsatz führend. Links nierenförmige grosse Perforation, stinkende Eiterung. Vorn über dem Processus brevis, zum Theil der oberen Gehörgangswand angehörend, eine hịrsekorngrosse Perforation. Ausspülnngen der Paukenhöhle mittelst des Katheters, des oberen Hohlraumes mittelst des Antrumröhrchens. Heilung nach 8 Monaten. Jetat ist die Perforation über dem Processus brevis durch eine feine Narbe geschlossen.

5. Wilhelm M., 29 Jahre, hat vor 3 Jahren schon an Polypen gelitten. Rechts Fötor. Polyp in der Gegend der Membrana Shrap- 
nelli durch Aetzen beseitigt. Es erscheint eine hirsekorngrosse Perforation, kein Perforationsgeräusch. Ausspülungen mit der Caniile. Heilung nach 8 Wochen mit narbigem Verschluss.

6. Wilhelm R., 44 Jahre, Schmied. Seit Jahren schwerhörig. Links in der Gegend der Membrana Shrapnelli Perforation, in beiden Paukenhöhlen Exsudat. Beiderseits Paracentese und Entleerung des Secretes. Aus der oberen Oeffnung wächst eine Granulation, welche auf Aetzen verschwindet. Antrumeanïle. Heilung nach 6 Wochen mit Narbenbildung.

7. Marie F., 23 Jahre, hat seit Jahren eine chronische Eiterung links. Seit 3 Wochen heftige Kopfschmerzen. Etwas hysterische Person. Uhr contact, $\mathrm{C}$ nach links, Flüstern 1 Meter. Links vorn oben herabkommend ein Polyp, stinkende Eiterung. Nach Abschnürung und Aetzung des Polypen zeigt sich eine nahe am Rivinischen Ausschnitt gelegene Perforation, aus welcher mit der Canizle Cholesteatommassen entfernt werden. Tubenverschluss. Nach täglichen Ausspîlungen mit dem Antrumröhrchen verliert sich der Fötor bald. Nach 10 Wochen hört die Eiterung auf. Die Perforation bleibt offen.

8. Auguste W., 28 Jahre, hat seit frühester Jugend öfters Ohrenzwang gehabt, seit 6 Jahren Ohrenlanfen, in den letzten Monaten Kopfschmerzen und Schwindel. Anämische Person. Rechts Residuen. Links Trommelfell verdickt, vorn oben, zum Theil im Knochen sitzend, eine hanfkorngrosse Perforation, aus welcher cholesteatomatöse Massen heraushängen. Perforationsgeränsch fehlt. Pankenhöhle frei von pathologischen Producten. Fötor. Ausspuilungen mit dem Antrumrölurchen fördern Cholesteatom zu Tage; nach einiger Zeit Aufhören des Fötor. Eine wegen der bestehenden Kopfschmerzen vorgenommene Aufmeisselung des Warzenfortsatzes führte zu keiner Communication mit der Paukenhöhle. Nach Verheilung der Wunde werden die Ausspülungèn mit dem Antrumröhrchen fortgesetzt. Durch Darreichung von Eisen verschwinden auch die Kopfschmerzen. Nach 10 Wočhen ist die Höhle dauernd trocken, Patientin ist sehr kräftig geworden.

9. August M., 31 Jahre. Seit Kindheit links Ohrenduss, stinkend. Grosse Perforation in den Knochen reichend und die Stelle der ganzen Membrana flaccida einnehmend. Das ganze Trommelfell fehlend bis auf 2 den Hammer am Margo tympanicus festhaltende Stränge. Ausspïlnngen durch die Tuba zur Behandlung der Paukenböhleneiterung und mit der Canïle zur Behandlung der Affection des oberen Raumes. Heilung der Eiterung im oberen Raum nach 7 Monaten.

Es lässt sich wohl annehmen, dass in diesen Fällen keine tiefgreifenden Knochenaffectionen vorhanden gewesen sind, da solche erfahrungsgemäss nicht durch eine so milde Therapie zur Heilung gelangen.

Die Heilung erfolgte, wie die Krankengeschichten lehren, entweder mit Narbenbildung oder mit bleibenden Perforationen und 
trat ein nach einer Behandlungsdauer, welche zwischen $3 \mathrm{Wo-}$ chen und $5 / 4$ Jahren schwankt.

Die andere Art, auf welche therapeutisch gegen den abgeschlossenen oberen Hohlraum vorzugehen wäre, würde in Masseninjectionen von der Tuba aus bestehen, nachdem vorher eine Communication mit der.Paukenhöhle hergestellt worden ist.

Es lässt sich dies erreichen durch Fortnahme einer der Wände, welche den oberen Hohlraum von der Paukenhöhle trennen. Da die membranösen Theile schnell wieder verwachsen wiirden, so wird es sich empfeblen, die von Knochen zusammengesetzten Wände zum Angriffspunkt zu nehmen. Am geeignetsten erweist sich die mediale Wand, an deren Bildung Hammer und Amboss betheiligt sind. Zur Entfernung des Hammers existiren wohl ausgebildete Methoden ${ }^{1}$ ). Nicht dasselbe lässt sich ïber Extraction des Amboss sagen. Seine Lage in dem fuir das Auge nicht mehr zugänglichen Raume der Paukenhöhle bringt es mit sich, dass die Anwendung von Instrumenten zu seiner Entfernung bedeutend erschwert wird. Kesse12) bahnt sich zor. Wegnahme des Amboss und Freilegung des Steigbügels den Weg dadurch, dass er einen Theil der oberen hinteren Gehörgangswand entfernt. Es hat aber dies Verfahren keine allgemeinere Anwendung gefunden. Ich habe zur Entfernung des Ambosses ein einfaches

Fig 11 (1).

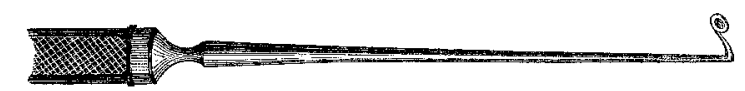

Instrument zur Entfernung des Amboss auf der linken Seite. ${ }^{3}$ )

Instrument construirt, dessen Abbildung obenstehend erfolgt (Fig. 11). Ich ging dabei von der Idee aus, dass es möglich sei, den Amboss nach Trennung rom Steigbügel und durch Wegnahme des Hammers durch einen von oben auf seinen kurzen Fortsatz wirkenden Hebel nach unten zu dislociren und in das Gesichtsfeld zu bringen. Ich wählte deshalb für die Länge des kurzen Schenkels die gerade Entfernung vọn der höchsten Höhe des Rivinisschen Ausschnittes bis zur Insertion des kurzen Amboss-

1) Schwartze, Lehrbuch. S. 283.

2) Ueber Ausschneiden des Trommelfells und Mobilisiren des Steigbügels. Oesterreich. ärztliche Vereins-Zeitung 1879.

3) Für die rechte Seite muss ein Instrument genommen werden, welches dem Spiegelbild des obigen entspricht. 
schenkels, und als Winkel denjenigen, welchen der Sporn der oberen Gehörgangswand nach der Paukenhöhle zu bildet. Wird das Instrument nun entlang der oberen Gehörgangswand eingeführt und, sobald der kurze Hebelarm in die Paukenhöhle gelangt ist, zurückgezogen, so dass der horizontale Theil gerade im Rivinischen Ausschnitt liegt, der kürzere winklige Theil in seiner ganzen Länge mit der Knochenniesche über dem Gehörgang Fühlung hat, so lässt sich der kleine Hebel durch eine einfache Rotation nach hinten auf der annähernd kreisbogenförmigen Fläche der Niesche (deren Centrum der höchste Punkt des Rivinischen Ausschnittes ist) über den kurzen Fortsatz des Amboss führen (vgl. Fig. 1). Der am Ende. des kurzen Schenkels befindliche Knopf drückt den Amboss nach unten und löst ihn aus seiner Gelenkverbindung. Mit der Pincette lässt sich dann leicht der in die Paukenhöhle fallende Knochen entfernen.

Durch vielfache Versuche an der Leiche konnte. ich mich von einer derartigen Wirkung des Instrumentes direct durch den Augenschein iiberzeugen, indem ich nach Wegnahme des Schädeldaches und Tegmen tympani von einem Collegen die Operation ausführen liess. Am Lebenden gelang die Extraction ebenfalls in einem Falle, wo wegen quälender Ohrgeräusche die Entfernung des Hammers und Amboss vorgenommen wurde.

Die Chorda wird gewöhnlich bei diesem Verfahren durch das Herabdrücken des Amboss gezerrt und erscheint im Gesichtsfeld als ein weisser Faden. Eine Zerreissung habe ich an der Leiche nicht constatiren können. Bei der Patientin, an der die Entfernung des Amboss vorgenommen wurde, trat Geschmackslähmung auf der betroffenen Seite ein, ohne jedoch die Kranke irgendwie zu incommodiren. 14 Tage nach der Operation bestand die Lähmung noch.

In einigen Fällen von Eiterung mit Perforation iuber dem Processus brevis, in welchen die Extraction des Amboss versucht wurde, gelang die Entfernung des Knochens nicht. Ich schreibe dies dem Umstande zu, 'das durch die Caries am Rivinischen Ausschnitt das Hypomochlion für den Hebel sich nicht mehr an der richtigen. Stelle befindet und dass das Instrument deshalb nicht in der beabsichtigten Weise wirken konnte. Ausserdem kann ja der Amboss auch schon cariös zerstört sein und ganz oder theilweise fehlen, oder wenigstens dislocirt sein. Jedenfalls ist ein Versuch zu seiner Entfernung, weil unschädlich, jedesmal zu unternehmen. Zur Durchtrennung des Ambosssteigbügel- 
gelenkes, welches der Entfernung des Amboss nothwendigerweise voraufzugehen hat, empfiehlt es sich, nach völliger Umschneidung des Trommelfells ein Tenotom ') mit nach oben gerichteter Schneide von hinten her immer unter Fühlung mit dem Hammergriff an diesem in die Höhe zu schieben und, sobald der Widerstand der Sehne des Tensor gefühlt wird, dieselbe durch eine kleine Hebelbewegung zu durchtrennen. Darauf wird das Instrument in derselben Ebene um eine halbe Wendung gedreht, bis Fühlung mit dem langen Ambossschenkel eintritt, und mit nach abwärts sehender Schneide die Gelenkverbindung mit dem Steigbügel durchschnitten.

Gelingt es nun, beide Gehörknöchelchen zu entfernen, so kann von 2 getrennten Räumen nicht mehr die Rede sein. Aber gesetzt auch, der Amboss bleibt zuritck, so wird dadurch nicht viel geschadet. Schon allein die Wegnahme des Hammers zerstört eigentlich den ganzen Raum, da mit ihm die ganze vordere, grösstentheils mediale und auch untere Wand beseitigt wird. Lässt sich der Amboss nicht entfernen, so bleibt höchstens noch ein Theil der unteren und eventuell ein Theil der hinteren Wand bestehen. Bei der Herausnahme des Hammers wird auch die sich kuppelförmig (Prussak l. c.) über die Tubenmündung spannende, zur Sehne des Tensor ziehende Falte zerstört, und es kann der Wasserstrom von der Tuba aus directer an den hochgelegenen Krankheitsherd dringen, während er bei Bestehen der Falte durch Anprall an diese nach hinten abgelenkt wird. Die Entfernung der Gehörknöchelchen ist nur als vorbereitende Operation aufufassen, wenn ja auch bei Caries derselben ihre Beseitigung schon an sich geboten sein wird. Sie soll es ermöglichen, dass die Spülflïssigkeit von der Tuba aus den erkrankten oberen Raum ordentlich treffen kann. Regelmässige Durchspülungen sind das Postulat für das Zustandekommen einer Heilung.

$\mathrm{Da}$, wie oben bereits auseinandergesetzt, bei der vorliegenden Form der Eiterung gewöhnlich der Rivinișche Ausschnitt und seine Umgebung cariös ist, und da auch Grund zu der Annahme vorliegt, dass die Caries sich an der Innenseite der Niesche über dem Gehörgang weiter erstreckt, so empfiehlt es sich, alle diese Theile mit dem scharfen Löffel $\mathrm{zu}$ bearbeiten, um alles Kranke thunlichst zu entfernen. Um an der Innenseite der late-

1) Vgl. Abb. bei Schwartze, 1. c. S. 275 . 
ralen Wand der Paukenhöhle möglichst hoch hinaufreichen zu können, bediene ich mich eines Löffels von geringer winkliger Knickung, wie ihn die Abbildung zeigt (Fig: 12). Freilich kann

Fig. $12\left(\frac{1}{1}\right)$.

Winkliger scharfer Löffel zum Abkratzen des Margo tympanicus.

man auch damit nicht alle die Theile erreichen; welche den Sitz der Caries abgeben können: Der Grund der Knochenniesche und das Tegmen tympani sind für das Instrument unzugänglich.

Folgende Fälle wurden in der beschriebenen Weise behandelt.

10. Carl M., 44 Jahre, hat vor 6 Jahren einen Polypen im rechten $\mathrm{Ohr}$ gehabt, nach dessen Entfernung völlige Heilung eingetreten sein soll. Seit 2 Jahren Ohrenlaufen, Pochen in demselben Ohr, Schwindelgefühl, öfters Schmerzen. Rechts über dem Processus brevis eine hirsekorngrosse Perforation, zum Theil der oberen Gehörgangswand angehörend. Am Ende des Hammergriffes eine zweite, grössere; starker Fötor. Uhr rechts beim Anlegen, Flüsterzahlen gar nicht gehört. C vom Scheitel nach rechts. Trommelfell und Hammer werden excidirt. Hammerkopf fast ganz fehlend. Tags darauf Kopfschmerz und Schwindel verschwunden. Tägliche Massendurchspülungen per tubam. Nach 8 Tagen Fötor beseitigt. Trommelfell in Regeneration begriffen. Gehộr wie zu Anfang. Patient mit dem Erfolg zufrieden verlässt die Behandlung.

11. Panl D., 22 Jahre, leidet seit Kindheit an linksseitigem Ohrenfluss. Seit 3 Monaten starke Kopfschmerzen, seit 8 Wochen Schwindel und Schwere im Kopf. Geringe Secretion, starker Fötor, Perforation von Stecknadelkopforösse am Rivinischen Ausschnitt.

Links Uhr $8 \mathrm{Cm}$.

Flüstern $40 \mathrm{Cm}$.

$\mathrm{C}$ nach links.

Rasseln in der Paukenhöhle, infolge dessen Paracentese mit dem Galvanokauter und Durchspülungen per tubam. Der Geruch bleibt bestehen, deshalb nach, 4 Wochen Aufmeisselung des Aritrum. Da keine Communication erreicht wurde, wird die Wunde nicht lange offen gehalten. Ausspülung der Höhle über dem kurzen Fortsatz mit dem Antrumröhrchen. Kopfsehmerzen und Geruch verschwinden nie ganz. Nach einer längeren Unterbrechung der Behandlung wird 5 Monate nach der Aufmeisselung der Hammer entfernt. Hammerkopf grösstentheils cariös zerstört; Margo tympanicus erweicht, mit dem scharfen Löffel abgekratzt. Nach 14 Tagen sind alle subjectiven Symptome verschwunden, kein Fötor, das Trommelfell in Regeneration begriffen, die Paukenschleimhaut succulent und wulstig. Da der 
Kranke sich den Katheter selbst einführen kann, wird er entlassen. Nach einem halben Jahr ist. das Trommelfell bis anf einen Defect hinten unten geschlossen. Oben ist das Trommelfell anscheinend mit der Labyrinthwand verwachsen, so dass der Eingang in die Höhle hinter dem Rivinischen Ausschnitt offen bleibt. Secretion nicht mehr wahrnehmbar, subjectives Wohlbefinden, kein Geruch.

Links Uhr $10 \mathrm{~cm}$.

Flüstern $50 \mathrm{Cm}$.

$\mathrm{C}$ nach links.

12. Friedrich B., 17 Jahre. Will erst seit 2 Jahren Ohreiterung haben. Links Caries. Rechts grosse nierenförmige Perforation, im Trommelfell iiber dem Processus brevis eine hirsekorngrosse Oeffnung, deren unterer Rand von. der Spitze des kurzen Fortsatzes um circa $2 \mathrm{Mm}$. entfernt ist. Die Sonde führt in einen sich hauptsächlich nach vorn und aussen erstreckenden Raum. Medialwärts stösst sie auf rauhen Knochen.

Rechts Uhr beim Anlegen.

Flüstern ins Ohr.

Kopfschmerz, Schwindel. Längere Anwendung des Antrumröhrchens ftihrt kein Aufhören des Geruches herbei. Hammerextraction. Amboss ist mit der Sonde nicht zu fühlen. Hammerkopf vorn und lateralwärts cariös zerfressen. Gelenkfläche erhalten. Rivinischer Ausschnitt erheblich erweicht, mit dem Löffel ausgeräumt. 2 Tage später Kopfschmerz und Schwindel verschwunden. Gehörgang etwas verschwollev. In den nächsten Tagen Aufhören des Fötor. Nach 6 Wochen ist die Höhle in ihren Wandungen ganz glatt, geringe Secretion. 4 Monate nach der Operation Höhle oben ganz trocken. Trommelfell nicht wieder gewachsen. Hinten unten in der Paukenhöhle alle 4 Tage ein Tröpfehen geruchlosen Eiters.

Uhr beim Anlegen.

Flüstern $10 \mathrm{Cm}$.

13. Willy H., 16 Jahre, leidet seit dem 2. Lebensjahre links an Ohrenlaufen. Im letzten Jahre stellten sich öfters Ohrenreissen, Schwindel, Kopfschmerz, Sausen ein, verbunden mit Hörverschlechterung auf der betroffenen Seite. Der Vater des Patienten ist wegen eines Ohrenleidens operirt worden. Links Trommelfell erhalten. Paukenhöhle frei, kein Perforationsgeräusch. In unmittelbarer Nähe des Rivinischen Ausschnittes eine gut stecknadelkopfgrosse Perforation, in welcher grane, schmierige Massen lagern. Fötor. Ausspülungen mit der Antrumcanüle bringen, Wochen lang angewendet, weder Nachlass der subjectiven Symptome, noch vermögen sie, trotzdem eine Menge krümligen Inhaltes entleert wird, ein Aufhören des Geruches zu bewirken. Deshalb wird der Hammer entfernt. Es zeigt sich die vordere äussere Seite des Kopfes zerfressen. Der Margo tympanicus erheblich erweicht, wird mit dem scharfen Löffel abgekratzt. Tägliche Durchspiilungen von der Tuba aus beseitigen in wenigen Tagen den Fötor und bringen die subjectiven Beschwerden zum Verschwinden. Dagegen beginnt die Schleimhaut der Paukenhöhle stark 
zu schwellen und überragt die Ränder des sich regenerirenden Trommelfells. Mehrfache Abtragungen mit der Schlinge und Aetzungen mit Lapis schränken die Wucherung allmählich ein. Nach 6 Monaten ist das Trommelfell bis auf die Partie am Rivinischen Ausschnitt und bis auf eine Liücke am Margo tympanicus im hinteren oberen Quadranten wieder gewachsen. Aus der letzteren ragen cholesteatomatöse Massen hervor. Da dieselben nach weiterer viermonatlicher Durchspülung per tubam nicht verschwinden, wird die Eröffnung des Warzenfortsatzes vorgenommen. In der ersten Zeit entleeren sich noch ziemlich viele derartiger Massen. Jetzt ist die Eiterung minimal, geruchlos und es steht baldige definitive Heilung zu erwarten.

Uhr $12 \mathrm{Cm}$.

Flïstern $80 \mathrm{Cm}$.

14. Ernst K., 7 Jahre, hat seit dem 3. Lebensjahre links eine nach Scharlach entstandene Eiterung. Vegetationen im Nasenrachenraum werden mit dem Trautmann'schen Löffel entfernt. Im Gehörgang ein Polyp von vorn oben ausgehend. Nach Entfernung desselben mittelst der Schlinge erscheint eine Perforation in der Nähe des Rivinischen Ausschnittes und eine zweite am hinteren Rand des Trommelfells. Gehör 3/4 Meter für Flüsterzahlen. Der Geruch bleibt bestehen trotz Durchspülungen per tubam. Abgang von Cholesteatommassen. Deshalb Hammerexcision unternommen. Der Knochen wird aus seiner Lage gelöst, jedoch gelingt es nicht, ihn mit der Schlinge zu fassen, da er aus dem Gesichtsfeld verschwunden ist. Evidement des Margo tympanicus, Aufmeisselung des Antrum, welches Cholesteatom enthält. Nach 1/4 Jahr Eiterung geheilt, Trommelfell durch Narbe ersetzt; Hammer nicht zu sehen. Gehör wie zu Anfang.

15. Minna 0., 11 Jahre, ist schwerhörig, so lange sie denken kann. Adenoide Wucherungen im Nasenrachenraum werden beseitigt. Rechts fast ganz taub. Links stecknadelkopfgrosse Perforation in der Membrana flaceida. Der Knochen der oberen Gehörgangswand scheint gesund. Anfangs kein Perforationsgeräusch. Später stellt sich dasselbe ein unter Austritt von käsigen, stinkenden Massen aus der Perforation. Links Flüstern $3 / 4$ Meter. Uhr unsicher. Durchspiulungen von der Tuba aus haben auf den Fötor keinen Einfluss. Deshalb wird, trotzdem das andere $\mathrm{Ohr}$ nahezu taub ist, doch zur Hammerextraction geschritten. Obwohl der Rivinische Ausschnitt, dem Auge nach zu urtheilen, gesund erschien, findet er sich doeh in hohem Grade erweicht und muss mit dem scharfen Löffel abgekratzt werden. Der Hammerkopf zeigte an seiner vorderen lateralen Seite theils Defecte, theils Knochenneubildungen. Die Entfernung des Amboss gelang nicht. In den ersten Tagen trat Verschwellung des Gehörgangs ein und erhebliche Herabsetzung des Gehörvermögens. Die Haut der oberen Gehörgangswand hängt wie eine Schürze herab; tägliche Durchspillungen von der Taba aus. Nach 2 Wochen hat sich die Haut wieder angelegt, der Gehörgang ist weit. Flüsterzahlen $30 \mathrm{Cm}$. Der Geruch ist nicht vollständig versehwunden. Nach wei- 
teren 3 Wochen ist das Hörvermögen wieder wie vor der Operation. Jedoch bleibt immer etwas Geruch. Es wird wohl nun auch hier zur Aufmeisselung geschritten werden müssen, da anzunehmen ist, dass die Ursache des Fötor im Antrum selbst oder dessen unmittelbarer Nähe, oder an Stellen, die der bisherigen Behandlungsart nicht zugänglich waren, ihren Sitz hat.

In den von Schwartze ${ }^{1}$ ) mitgetheilten Krankengeschichten von Hammerexcision betreffen die ersten 3 ebenfalls Individuen mit Perforation ïber dem Processus brevis. Sie wurden nach Entfernung des stets cariösen Hammers mit Durchspülungen von der Tuba aus behandelt und geheilt.

Wenn nach diesen Krankengeschichten nicht bezweifelt werden kann, dass die Entfernung des Hammers verbunden mit Evidement des Margo tympanieus zur Heilung der Eiterungen im oberen Raume der Paukenhöhle von grosser Bedeutung ist, die subjectiven Beschwerden und gewöhnlich auch den Fötor mit Sicherheit beseitigt, so drängt sich dabei doch die Frage auf: „Ist die Hammerextraction ein für den Kranken ganz gleichgültiger Eingriff?" Quoad vitam dürfte die Frage unbedingt zu bejahen sein, da ungïnstige Ausgänge in dieser Beziehung bisher nicht beobachtet sind. Untersuchen wir aber, wie es sich quoad functionem verhält. Wenn wir wüssten, wie gross das Hörvermögen eines normalen Ohres nach Entfernung des Hammers sein wïrde, so wäre dadurch eine Grenze bestimmt, bis za welcher mit der Vornahme der Operation unter Berücksichtigung des jeweiligen Hörvermögens des erkrankten Ohres ohne Bedenken gegangen werden könnte. Da wir aber diese Kenntniss nicht besitzen, so können wir nur aus dem vorhandenen Krankenmaterial eine relative Bestimmung über das Hörvermögen eines Ohres bei fehlendem Hammer treffen.

In dem 2. der Schwartze'schen Fälle wurden noch Flüsterworte auf $50 \mathrm{Cm}$, in einem der unsrigen sogar noch auf $80 \mathrm{Cm}$. nach Entfernung des Hammers wahrgenommen. Es würde demnach nicht bedenklich sein, die Hammerexcision bei einem Kranken, welcher Flüsterzahlen bis auf $80 \mathrm{Cm}$. hört, vorzunehmen, um so weniger, als sich, wie unsere Fälle lehren, eine dauernde Hörverschlechterung (abgesehen von einer in den ersten Tagen auftretenden, dann aber wieder verschwindenden) nach Wegnahme des Knochens nicht gezeigt hat. Diese Beobachtung konnten wir auch noch an einer Anzahl solcher Individuen machen,

1) Lehrbuch. S. 287. 
bei welchen der Hammer aus anderen Ursachen entfernt werden musste. Ist das Gehör auf dem nicht betroffenen $\mathrm{Ohr}$ noch gut, so wird man sich auch entschliessen können, die Operation bei einem Hörvermögen, welches die oben gefundene Grenze noch ïberschreitet, vorzunehmen, selbst auf die Gefahr hin, dass eine Beeinträchtigung der Function dadurch eintreten könnte. Ist dagegen das zu operirende $\mathrm{Obr}$ das bessere oder gar das allein hörende, so soll die Operation nur nuternommen werden, wenn sich alle anderen Heilversuche als vergeblich herausgestellt haben. In dieser Lage befanden wir uns in dem letztcitirten Falle.

Die Regeneration des Trommelfells wirkt nicht störend auf die Behandlung mit Masseninjectionen durch die Tuba. Im Gegentheil kann es eher von Vortheil sein, wenn der Wasserabfluss dureh Verengerung der Abflussöffnung etwas behindert wird. Die Flüssigkeit kann dann nicht so schnell und leicht abfliessen und wird deshalb die erkrankten Theile länger und energischer bespülen. So lange noch Caries am Margo tympanicus oder dessen nächster Umgebung vorhanden ist, schliesst sich das neugebildete Trommelfell nie ganz, sondern bleibt in der Nähe des erkrankten Knochens offen und ermöglicht so eine fortgesetzte Anwendung der Durchspülungen von der Tuba aus. Nicht ganz selten kommt es nach der Extraction des Hammers zu einer Entzündung der vorher ganz gesunden Paukenhöhle, vielleicht durch Infection mit dem im oberen Raum befindlichen zersetzten Secrete. Diese Entzündung führt gewöhnlich zu starker Wulstung der Schleimhaut, welche die Abtragung mit der Schlinge und die Anwendung von Aetzmitteln erfordert (Fall 12,14). Der Zustand ist ein sehr hartnäckiger und erstreckt sich oft auf Monate, weicht aber regelmässig einer geeigneten Therapie.

Erfolgt nun bei der geschilderten Behandlung keine Heilung der Eiterung, so hat dies seinen Grund darin, dass noch Herde vorhanden sind, welche von den bisherigen therapeutischen Maassnahmen unbeeinflusst blieben. In der Regel wird es sich in solchen Fällen um Processe im Antrum mastoideum handeln. Wie leicht dasselbe mit befallen wird, oder werden kann, sahen wir bereits weiter oben. Durch Erfahrung wissen wir, dass cariöse Processe im Warzenfortsatz, oder selbst nur einfache Retentionen von Eiter mit Zersetzung vermittelst Durehspülungen der Pauke von der Tuba aus wenig oder gar nicht beeinflusst werden, und deshalb führt gerade der Misserfolg der letzteren Therapie zur 
Annahme einer Erkrankung des Warzenfortsatzes. Im Fall 14 machte sich das Vorhandensein einer solchen Complication bemerklich durch Persistiren einer Perforation am hinteren oberen Rande des Trommelfells nach erfolgter Regeneration der Membran. Bekanntlich sind Perforationen an dieser Stelle eventuell complicirt mit durchwachsenden Granulationen, ein Zeichen für Caries des Warzenfortsatzes. Sind wir also zu der Ueberzeugung gelangt, dass neben der Erkrankung im Hammer-Amboss-Schuppenraum noch eine solche des Warzenfortsatzes vorliegt, so bleibt nichts übrig, als zu einer Eröffnung desselben zu schreiten, welche einmal die Wegnahme erkrankter Knochentheile ermöglicht, dann aber zur grïndlichen Durchspülung des Antrum, der Pauke und des erwähnten Raumes wesentlich beiträgt.

Die Aufmeisselung kann sich der Entfernung der Gehörknöchelchen sofort anschliessen, wenn sichere Zeichen für Erkrankung des Processus vorliegen (Fall 15), oder sie wird erst längere Zeit nach der Excision vorgenommen (Fall 14), wenn anlässlich der Erfolglosigkeit der bisherigen Therapie eine Erkrankung des Warzenfortsatzes für wahrscheinlich gelten muss, oder wenn sich Anzeichen einstellen, welche die Erkrankung zur Gewissheit machen. Aber auch wenn der Warzenfortsatz selbst gar nicht erkrankt ist, so wird durch seine Eröffnung doch ein Weg zur wirksamen Bespülung der Paukenhöhle geschaffen, besonders jener Theile, welche, wie wir annehmen müssen, bei Erfolglosigkeit der Extraction der Gehörknöchelchen und Evidement des Margo Sitz der Erkrankung sind: Tegmen tympani und Grund der Knochenniesche. Die Aufmeisselung des Warzenfortsatzes wird nur dann von Erfolg begleitet sein, wenn zuvor die Gehörknöchelchen entfernt und der Hammer-Amboss-Schuppenraum dadurch eröffnet wurde. Unterbleibt diese vorbereitende Operation, so ist auch eine Einwirkung auf die dem oberen Hohlraume angehörigen Theile Tegmen und Grund der Knochenniesche, durch Eröffnung des Antrum mastoideum sehr erschwert oder völlig ausgeschlossen, je nachdem Communication des Hammer-AmbossSchuppenraums mit der Paukenhöhle besteht oder nicht.

Die Scala der Behandlang von Eiterungen mit Perforation über dem kurzen Hammerfortsatz besteht demnach in: „desinficirenden Ausspülungen; Masseninjectionen per tubam; Entfernung der Gehörknöchelchen, Hammer und Amboss, verbunden mit Evidement des Margo tympanicus und Durchspülungen von der Tuba, schliesslich Eröffnung des Warzenfortsatzes." 
Aus der Wirksamkeit der einzelnen Methoden wird man nachträglich einen Schluss auf die Intensität und Extensität des jedesmaligen Krankheitsprocesses machen können.

Fassen wir noch einmal kurz die gewonnenen Resultate zusammen: Die zur Perforation über dem Processus brevis führenden Eiterungen haben ihren Sitz in einem zwischen Hammerkopf und Ambosskörper einerseits, Schläfenschuppe andererseits gelegenen, von der ïbrigen Paukenhöhle ganz oder grösstentheils abgeschlossenen Hohlraum. Sie führen gewöhnlich zu Caries der beiden äusseren Gehörknöchelchen und des Margo tympanicus und sind häufig complicirt mit Polypen und Cholesteatombildung.

Bei den Fällen, wo ein breites Perforationsgeräusch auf eine genügende Durchgängigkeit schliessen lässt, sind Massendurchspülungen von der Tuba aus anzuwenden, fehlt das Perforationsgeräusch, Ansspülungen mit dem in die Perforation einzuführenden Antrumröhrehen. Findet bei dieser Behandlungsweise nicht innerhalb 8 Tagen ein Aufhören des Fötors und ein Nachlassen der subjectiven Beschwerden statt, so ist die Entfernung des Hammers und wo möglich auch des Amboss, sowie das Evidement des Margo tympanicus indicirt, mit nachfolgenden Durchspiilungen per tubam. Bleibt auch danach noch der Fötor bestehen, so muss die Eröffnung des Warzenfortsatzes vorgenommen werden. 